\title{
FIVE-YEAR WILKINSON MICROWAVE ANISOTROPY PROBE* OBSERVATIONS: GALACTIC FOREGROUND EMISSION
}

\author{
B. Gold ${ }^{1}$, C. L. Bennett ${ }^{1}$, R. S. Hill ${ }^{2}$, G. Hinshaw ${ }^{3}$, N. Odegard ${ }^{2}$, L. Page ${ }^{4}$, D. N. Spergel ${ }^{5,6}$ J. L. Weiland ${ }^{2}$,

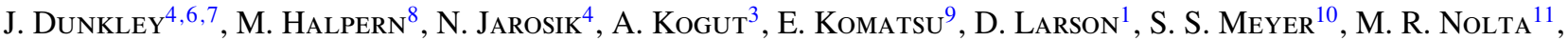 \\ E. Wollack ${ }^{3}$, AND E. L. Wright ${ }^{12}$ \\ ${ }^{1}$ Department of Physics and Astronomy, The Johns Hopkins University, 3400 N. Charles St., Baltimore, MD 21218-2686, USA; bgold@pha.jhu.edu \\ 2 Adnet Systems, Inc., 7515 Mission Dr., Suite A1C1 Lanham, Maryland 20706 \\ ${ }^{3}$ Code 665, NASA/Goddard Space Flight Center, Greenbelt, MD 20771, USA \\ ${ }^{4}$ Department of Physics, Jadwin Hall, Princeton University, Princeton, NJ 08544-0708, USA \\ ${ }^{5}$ Department of Astrophysical Sciences, Peyton Hall, Princeton University, Princeton, NJ 08544-1001, USA \\ ${ }^{6}$ Princeton Center for Theoretical Physics, Princeton University, Princeton, NJ 08544, USA \\ ${ }^{7}$ Astrophysics, University of Oxford, Keble Road, Oxford, OX1 3RH, UK \\ ${ }^{8}$ Department of Physics and Astronomy, University of British Columbia, Vancouver, BC V6T 1Z1, Canada \\ ${ }^{9}$ Department of Astronomy, University of Texas, Austin, 2511 Speedway, RLM 15.306, Austin, TX 78712, USA \\ ${ }_{10}$ Departments of Astrophysics and Physics, KICP and EFI, University of Chicago, Chicago, IL 60637, USA \\ ${ }^{11}$ Canadian Institute for Theoretical Astrophysics, 60 St. George St, University of Toronto, Toronto, ON M5S 3H8, Canada \\ ${ }^{12}$ PAB 3-909, UCLA Physics and Astronomy, P.O. Box 951547, Los Angeles, CA 90095-1547, USA \\ Received 2008 March 4; accepted 2008 June 19; published 2009 February 11
}

\begin{abstract}
We present a new estimate of foreground emission in the Wilkinson Microwave Anisotropy Probe (WMAP) data, using a Markov chain Monte Carlo method. The new technique delivers maps of each foreground component for a variety of foreground models with estimates of the uncertainty of each foreground component, and it provides an overall goodness-of-fit estimate. The resulting foreground maps are in broad agreement with those from previous techniques used both within the collaboration and by other authors. We find that for WMAP data, a simple model with power-law synchrotron, free-free, and thermal dust components fits $90 \%$ of the sky with a reduced $\chi_{v}^{2}$ of 1.14 . However, the model does not work well inside the Galactic plane. The addition of either synchrotron steepening or a modified spinning dust model improves the fit. This component may account for up to $14 \%$ of the total flux at the $\mathrm{Ka}$ band $(33 \mathrm{GHz})$. We find no evidence for foreground contamination of the cosmic microwave background temperature map in the $85 \%$ of the sky used for cosmological analysis.
\end{abstract}

Key words: cosmic microwave background - cosmology: observations - diffuse radiation - Galaxy: halo Galaxy: structure - ISM: structure

\section{INTRODUCTION}

The Wilkinson Microwave Anisotropy Probe (WMAP) produces temperature and linear polarization radio maps at five frequencies with $1^{\circ}$ or better resolution and tightly constrained systematic errors. The frequency bands are centered on 22, 33, 41, 61, and $94 \mathrm{GHz}$, denoted K, Ka, Q, V, and W, respectively (see Page et al. 2003 for details). While designed to measure the cosmic microwave background (CMB) radiation, it also observes the large-scale structure of our Galaxy at angular scales and frequencies that are relatively unexplored. Study of our own Galaxy has had a significant effect on our understanding of galaxies in general.

Radio emission from galaxies is generally understood as arising from three effects: "nonthermal" synchrotron emission from relativistic electrons spiraling in large-scale magnetic fields, "thermal" free-free emission from nonrelativistic electron-ion interactions, and emission from vibrational modes of thermal dust grains. At lower radio frequencies, the synchrotron emission is usually dominant, with flux decreasing at higher frequen-

\footnotetext{
* WMAP is the result of a partnership between Princeton University and NASA's Goddard Space Flight Center. Scientific guidance is provided by the WMAP Science Team.
}

cies approximately according to a power $\operatorname{law}^{13}(\beta \approx-3)$. Freefree emission has a flux that is nearly constant with frequency ( $\beta \approx-2.1$ ), so free-free emission becomes relatively more important than synchrotron at higher frequencies. Typically, the crossover frequency is near $60 \mathrm{GHz}$ at higher latitudes, but can be $20 \mathrm{GHz}$ or lower in specific regions in the Galactic plane. Frequencies above $\sim 60 \mathrm{GHz}$ begin to probe the tail $(\beta \approx 2)$ of vibrational dust emission, which is dominant around $90 \mathrm{GHz}$. In addition to these three foregrounds, much recent work has focused on the possibility of significant emission from rapidly rotating dust grains; this emission is thought to peak somewhere in the $10-30 \mathrm{GHz}$ range and fall off roughly exponentially at higher frequencies.

The spectral behavior for diffuse foregrounds is of great interest. The spectrum for synchrotron radiation follows the energy distribution for high-energy electrons, which is not a pure power law. The highest energy electrons lose energy more quickly and thus are reduced in regions where they have not been replenished. Such energy loss shows up as a gradual steepening $(d \beta / d v<0)$ in the power-law index by about 0.5 at

\footnotetext{
13 In this paper, we use the notation that flux density is $S \sim \nu^{\alpha}$ and antenna temperature is $T \sim v^{\beta}$, with the spectral indices related by $\beta=\alpha-2$. Unless otherwise noted, results will be expressed in antenna temperature. For the most commonly used pixel size in this work $(0.92 \times 0.92)$, the conversion from antenna temperature to flux is approximately $4.0(v / 22.5 \mathrm{GHz})^{2} \mathrm{Jy} \mathrm{mK}^{-1}$ (Page et al. 2003).
} 
frequencies above 10-100 MHz. Further, while the overall index as extrapolated from lower frequencies is $\beta \approx-2.7$ (Reich \& Reich 1988; Lawson et al. 1987; Reich et al. 2004), higher frequencies may preferentially sample more energetic electron populations and thus have a flatter index $(\beta \approx-2.5$; Bennett et al. 2003). Observations of both discrete sources (Green 1988; Green \& Scheuer 1992) and external galaxies (Hummel et al. 1991) show a wide variety of synchrotron behavior. Free-free emission also does not follow a strict power law, but the physics is well understood and the variation of the power-law index over WMAP's bands is so small that it can be neglected. Finally, the Rayleigh-Jeans tail for vibrational dust emission (i.e., below $100 \mathrm{GHz}$ ) has never before been accurately measured and the relevant material properties of the dust grains themselves are not fully understood (Agladze et al. 1994; Meny et al. 2007).

The main focus in this work is on foreground emission. Section 2 describes updates to masks and foreground-fitting procedures used in previous WMAP analyses (Bennett et al. 2003; Hinshaw et al. 2007). A new method to explicitly marginalize over foregrounds for the low multipole analysis is described in a companion paper (Dunkley et al. 2009). A new fitting process is described in Section 3, which has the following features.

1. The fitting is entirely in real space with no spherical harmonic decomposition for any component.

2. The spectral indices of the synchrotron and dust emission are not generally assumed to be constant and are allowed to vary down to the scale of the fit (approximately $1 \mathrm{deg}^{2}$ ).

3. The fit includes the CMB and automatically generates the full likelihood (including covariance) for all foreground parameters.

4. The polarization data are included and fitted simultaneously with the total intensity data.

This is similar to the technique of Eriksen et al. (2008); however, we fit the CMB in pixel space, use less smoothing on the maps, and attempt to obtain more information about individual foregrounds.

The results of the fit are described in Section 4. While the fitting technique used here delivers a CMB map with error bars, the map itself has not proved to be any better for cosmological analysis and so far has been used only as a check. Implications of the fit are discussed in Section 5. WMAP's cosmological results do not depend on the fitting process used here.

\section{5 YEAR FOREGROUND FITS}

\subsection{Masks}

The diffuse foreground masks are updated for the 5 year data analysis. The primary reason is to mask out free-free emission in the areas of the Gum Nebula and $\rho$ Oph, while keeping a simple method that applies to the whole sky rather than being ad hoc for these regions.

The new masks are based on 3 year public WMAP data products, ${ }^{14}$ specifically the 3 year $\mathrm{K}$ - and Q-band-average maps smoothed to $1^{\circ}$ resolution. These maps are converted to foreground-only maps by subtracting the 3 year Internal Linear Combination (ILC) map. A cumulative histogram is made of the

\footnotetext{
14 The new masks were based on 3 year data because they were needed before the 5 year maps could be finalized. The masks are made from flux cuts at high signal to noise on smoothed maps; thus, the difference between basing the masks on 3 year versus 5 year data is minimal. This was explicitly verified once the 5 year maps were finalized.
}
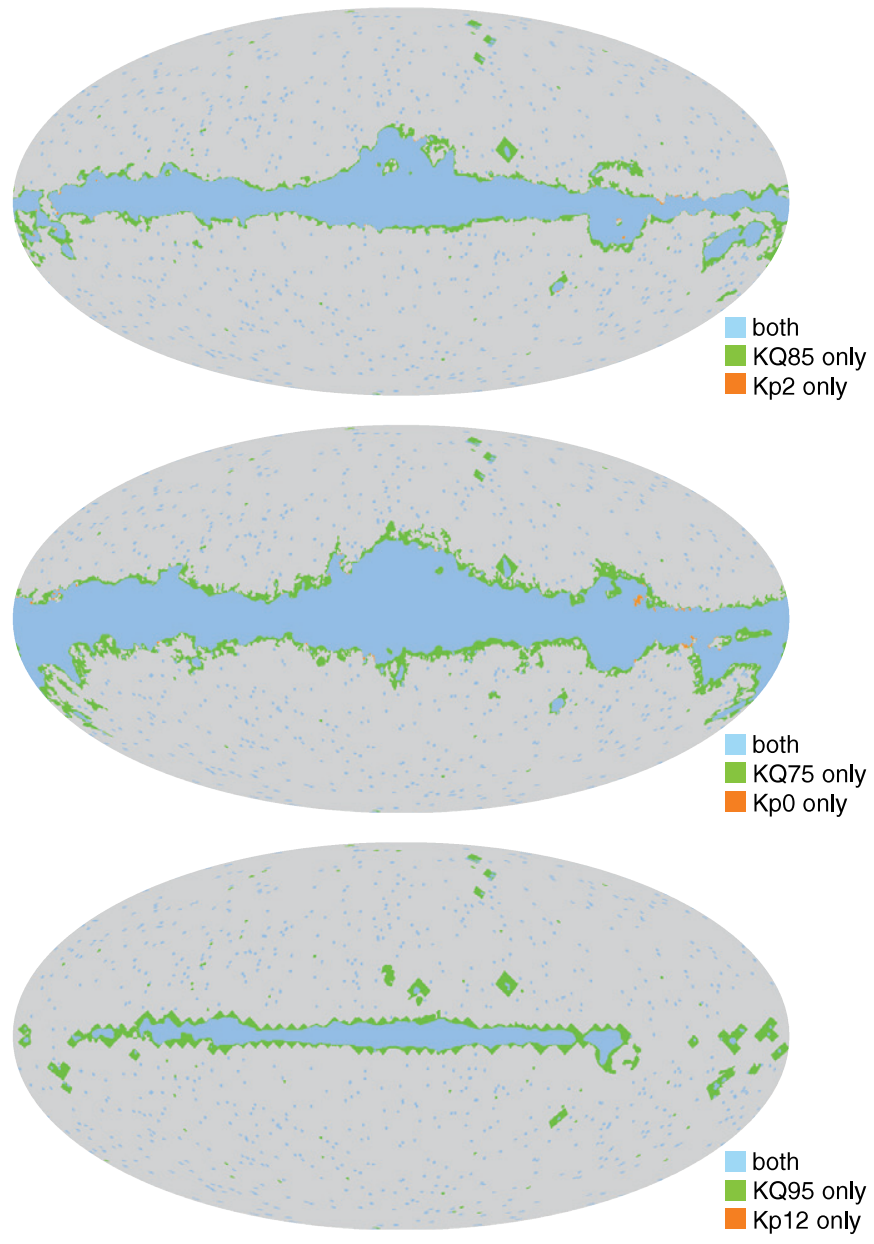

Figure 1. Comparison maps of the 5 year masks vs. the 3 year masks. The new masks cover slightly more of the Galactic plane and cover more regions with low synchrotron but high free-free emission. The diamond-shaped features arise because the new processing mask has been defined to correspond to lowresolution $\left(N_{\text {side }}=16\right)$ pixels, so that the same processing mask can be used at all resolutions. Top: comparison of KQ85 with the 3 year Kp2 mask. Middle: comparison of KQ75 with the 3 year Kp0 mask. Bottom: comparison of KQ95 with the 3 year Kp12 mask.

pixels in each foreground map, which serves as a lookup table to find a flux level used to define a cut over the desired percentage of the sky.

Cuts are made at intervals of $5 \%$ in the proportion of sky admitted by the resulting mask. The K- and Q-band cuts at each percentage level are combined. Resulting masks are inspected and compared with the masks used in the 1 and 3 year WMAP data analyses. We replace the old Kp2 mask with the combined $\mathrm{K}$ and $\mathrm{Q} 85 \%$ masks. This is the nominal mask for temperature data analysis and is denoted KQ85. We replace the old Kp0 mask with the combined $\mathrm{K}$ and Q 75\% masks (KQ75).

Each of the chosen masks is further processed by omitting any masked "islands" containing fewer than 500 pixels at HEALPix (Gorski et al. 2005) $N_{\text {side }}$ of 512. Each mask is then combined with a point-source mask, which has been updated from that described in Bennett et al. (2003) and Hinshaw et al. (2007) to include 32 newly detected sources from a preliminary version of the WMAP 5 year point-source catalog. Six sources in the final 5 year catalog are not included; these are relatively weak, with fluxes of $1 \mathrm{Jy}$ or lower in all WMAP bands. The last step combines each mask with the 5 year processing cut used to omit the Galactic plane from the mapmaking. A comparison of old and new masks is shown in Figure 1. 


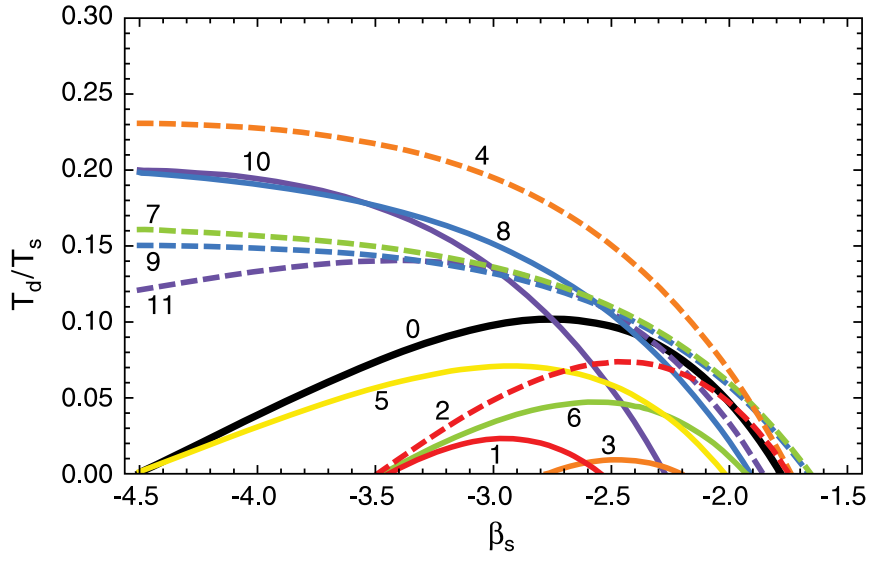

Figure 2. Slice in parameter space of the surface nulled by the ILC coefficients, assuming a three-component foreground model with power-law spectral behavior, $T(v)=T_{s} v^{\beta_{s}}+T_{f} v^{2.14}+T_{d} v^{\beta_{d}}$. Each line is for a single ILC region, denoted by a number. The parameter space is $T_{f} / T_{s}, T_{d} / T_{s}, \beta_{s}, \beta_{d}$. For this plot, the $x$-axis is $\beta_{s}$ and the $y$-axis is $T_{d} / T_{s}$. The parameters $T_{f} / T_{s}$ and $\beta_{d}$ are fixed at 0.7 and 1.8, respectively. Each color is a different ILC region. Despite the variety amongst ILC coefficients, they often null similar regions of parameter space.

The 3 year polarization mask was based on a cut in K-band polarized intensity combined with a model of the dust component (Page et al. 2007). The 5 year polarization analysis mask is the same as the 3 year version, with the exception that it is combined with the 5 year processing cut.

The Markov chain Monte Carlo (MCMC) fit described below uses a version of the combined $\mathrm{K}$ and Q 95\% mask (denoted KQ95, and which is similar to the old Kp12 mask) to distinguish "inside" from "outside" the Galactic plane. The mask was enlarged to account for smoothing, leaving approximately $91 \%$ of the sky.

\subsection{ILC Method}

The ILC method is used to produce a CMB map that is independent of both external data and assumptions about foreground emission. By construction, it leaves unchanged the component that has the spectrum of the $\mathrm{CMB}$ and acts as a foreground fit by filtering out the combined spectral shape that causes the most variance in the data. As a minimum variance method, the ILC is guaranteed to produce a map with good statistical properties, but the level of remaining contamination can be difficult to assess.

The algorithm used to compute the WMAP 5 year ILC map is the same as that described in the 3 year analysis (Hinshaw et al. 2007). We retain the same number of regional subdivisions of the sky and their spatial boundaries remain unchanged from the previous definitions. The frequency weights for each region are somewhat different, however, reflecting the 5 year updates to the calibration and beams. The new ILC regional coefficients are presented in Table 1, and the map itself is available on the LAMBDA Web site. ${ }^{15}$ The coefficients describe a filter that nulls certain spectral shapes. A slice in parameter space of the spectra nulled by the ILC is shown in Figure 2. Differences between new CMB maps and those from the 3 year release are further discussed in Section 5.

\footnotetext{
15 http://lambda.gsfc.nasa.gov/
}

Table 1

ILC Coefficients Per Region ${ }^{\mathrm{a}}$

\begin{tabular}{lrrrrr}
\hline \hline Region & K-band & Ka-band & Q-band & V-band & W-band \\
\hline 0 & 0.1336 & -0.6457 & -0.3768 & 2.2940 & -0.4051 \\
1 & -0.0610 & -0.1327 & -0.1873 & 1.7691 & -0.3880 \\
2 & 0.0037 & -0.2432 & -0.3792 & 1.7956 & -0.1768 \\
3 & -0.1104 & 0.2395 & -0.6424 & 1.5032 & 0.0101 \\
4 & -0.0843 & 0.1271 & -0.4584 & 0.9739 & 0.4417 \\
5 & 0.1918 & -0.7238 & -0.4902 & 2.4844 & -0.4622 \\
6 & -0.1052 & 0.2614 & -0.6223 & 1.0253 & 0.4407 \\
7 & 0.0913 & -0.3849 & -0.6033 & 2.3288 & -0.4319 \\
8 & 0.2208 & -0.5436 & -1.0938 & 3.2084 & -0.7918 \\
9 & -0.0922 & -0.0695 & -0.1810 & 1.2619 & 0.0808 \\
10 & 0.1724 & -0.9608 & 0.0350 & 2.6456 & -0.8923 \\
11 & 0.2374 & -0.8975 & -0.4897 & 2.7246 & -0.5747 \\
\hline
\end{tabular}

Note. ${ }^{\text {a }}$ The ILC temperature (in thermodynamic units) at pixel $p$ of region $n$ is $T_{n}(p)=\sum_{i=1}^{5} \zeta_{n, i} T^{i}(p)$, where $\zeta$ are the coefficients above and the sum is over WMAP's frequency bands.

\subsection{Maximum Entropy Method}

The maximum entropy method (MEM) is a spatial and spectral fit using templates that are intended to distinguish different low-frequency emission sources. By design, the MEM reverts to templates made from external data where WMAP's signal is low. One of the main goals for the MEM was to use high-signal regions to investigate the spectral properties of the foregrounds. The error properties for MEM maps are complicated and the model is essentially underconstrained so there is no meaningful goodness-of-fit statistic. The MEM maps were not used for analysis of the CMB itself.

The 5 year MEM analysis is largely unchanged from the 3 year analysis (Hinshaw et al. 2007). As before, the analysis is done on sky maps smoothed to a common resolution of $1^{\circ} \mathrm{FWHM}$ in all bands. To improve the signal-to-noise ratio $(\mathrm{S} / \mathrm{N})$, we now use maps degraded to HEALPix $N_{\text {side }}=128$ pixelization instead of $N_{\text {side }}=256$ (the pixel size for the former is 0.46 ). In the first year and 3 year analyses, the logarithmic term that forces the solution to converge to the priors for low $\mathrm{S} / \mathrm{N}$ pixels was missing a factor of $e$ (Cornwell et al. 1999); this has been fixed. The model is fit for each pixel $p$ by minimizing the functional $H=A+\lambda B$ (Press et al. 1992), where $A$ is the standard $\chi^{2}$ of the model fit, and we now use $B=\sum_{c} T_{c}(p) \ln \left[T_{c}(p) /\left(e P_{c}(p)\right)\right]$. Here, $T_{c}(p)$ is the model brightness of the emission component $c$ (synchrotron, free-free, dust) in pixel $p$ and $P_{c}(p)$ is the prior estimate of $T_{c}(p)$. The parameter $\lambda$ controls the relative weight of $A$ (the data) and $B$ (the prior information) in the fit. An iterative procedure is followed that uses residuals from the fit at each iteration to adjust the spectrum of the synchrotron component for each pixel. The MEM procedure was run for 11 iterations before stopping, the same as in the 3 year analysis.

The dust and free-free spectrum coefficients are required to follow power laws, with $\beta=+2$ for dust and $\beta=-2.14$ for free-free. Hence, any "anomalous" component, such as electric dipole emission from spinning dust, will be included in the synchrotron component. The priors used are also unchanged, using the Haslam $408 \mathrm{MHz}$ map (Lawson et al. 1987) for the synchrotron map, extinction-corrected H $\alpha$ (Finkbeiner 2003) for the free-free map, and Model 8 of Finkbeiner et al. (1999) for the dust map. The MEM maps are available for public download on the LAMBDA Web site. Figure 3 shows a comparison of the 5 year and 3 year MEM foregrounds, and the spectrum 

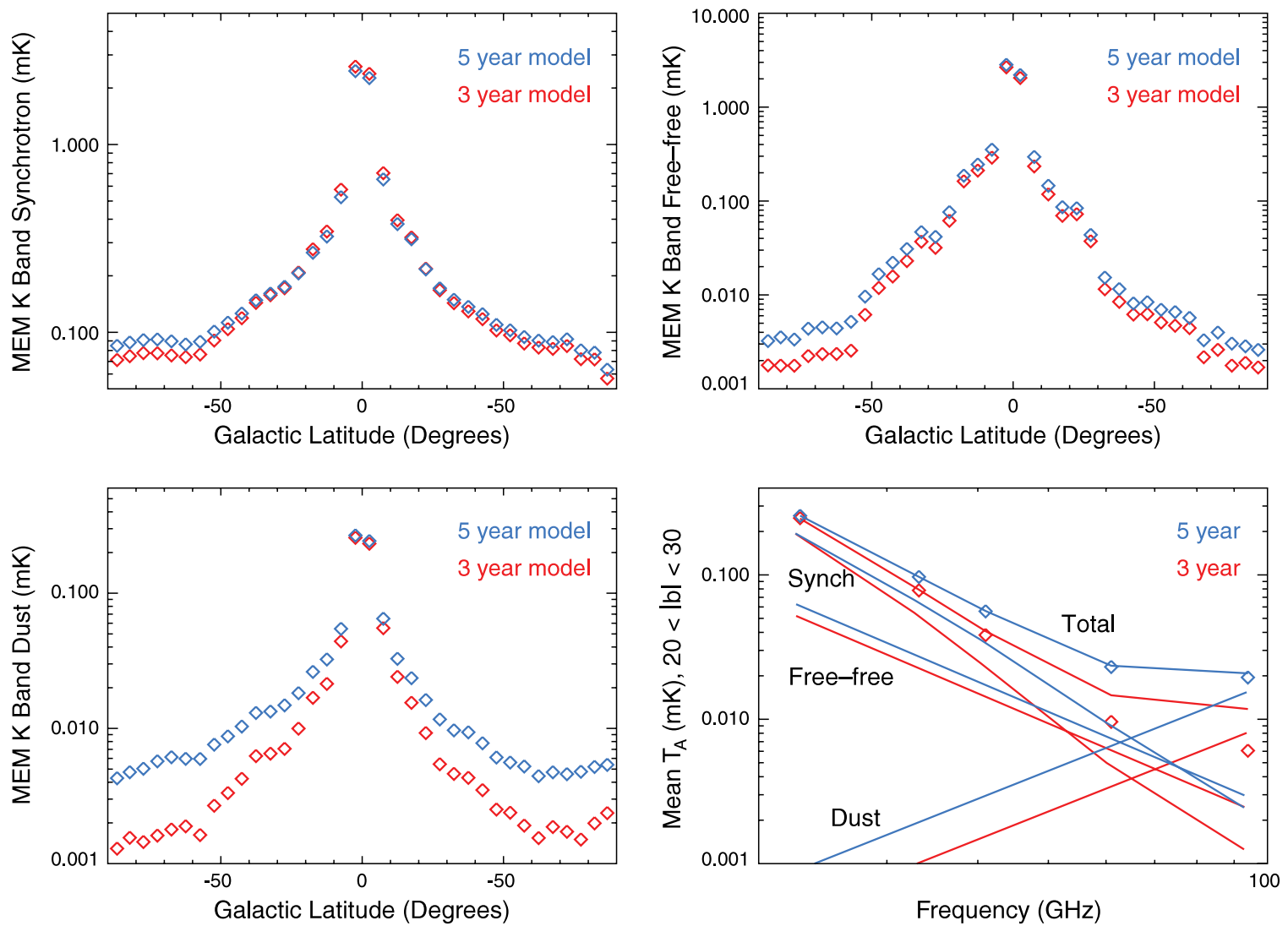

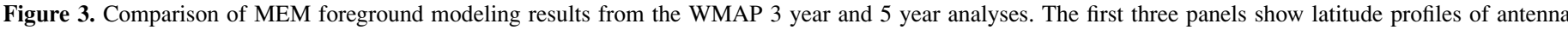

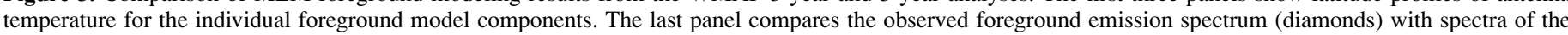

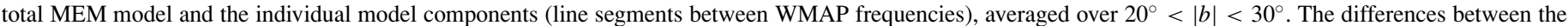

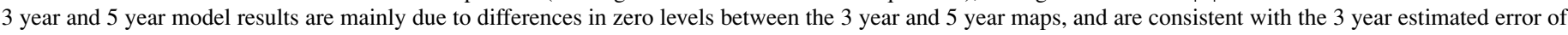

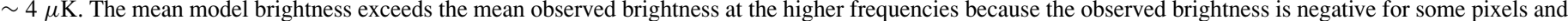
the model is constrained to be positive for each pixel. This is less apparent in the 5 year results because there are fewer negative pixels.

of components is compared to the total observed foreground spectrum for $20^{\circ}<|b|<30^{\circ}$.

Comparison of MEM results from the 5 year and 3 year analyses shows an increase in the model brightness of all foreground components at high Galactic latitudes. The changes are mostly due to differences in the zero levels of the 5 year and 3 year maps. The inclusion of the factor of $e$ in the MEM functional also leads to a small contribution. The method of setting map zero levels has not changed since the first-year analysis. The ILC CMB map is subtracted from the $1^{\circ}$ smoothed map in each frequency band, and the zero level is set such that a fit to the residual map of the form $T(|b|)=T_{p} \csc |b|+c$, over the range $-90^{\circ}<b<-15^{\circ}$, yields $c=0$ (Bennett et al. 2003). The 3 year analysis procedure was done using a preliminary 3 year ILC map in which the monopole was nonzero. Offsets of $21.1,19.4,19.3,19.4$, and $19.6 \mu \mathrm{K}$ should be added to the 3 year K-, Ka-, Q-, V-, and W-band maps, respectively, to give maps that yield csc $|b|$ fit intercepts of zero when the final 3 year ILC map is subtracted.

Available foreground templates are expected to trace the distribution of foreground emission more reliably than a csc $|b|$ model, so template fitting has been done to check the zero levels of the 5 year maps. Because the MEM is itself a template fit, this is essentially equivalent to fitting for the zero levels within the MEM procedure. The 5 year ILC map was subtracted from the 5 year $1^{\circ}$ smoothed maps, and the residual map for each band was fit to a linear combination of synchrotron, free-free, and dust templates plus a constant offset. Uncertainties in the zero levels of the templates were propagated to obtain an uncertainty in the derived offset value. For the synchrotron template, the $408 \mathrm{MHz}$ map of Haslam et al. (1982) was used with an offset of $5.9 \mathrm{~K}$ subtracted (Lawson et al. 1987). The quoted zero-level uncertainty of this map is $\pm 3 \mathrm{~K}$ (Haslam et al. 1982). For the free-free template, the composite all-sky $\mathrm{H} \alpha$ map of Finkbeiner (2003) was used, with a correction for extinction (using the dust extinction map of Schlegel et al. 1998) assuming that the dust is coextensive with the emitting gas along each line of sight (LOS; Bennett et al. 2003). The adopted zero-level uncertainty is \pm 1 Rayleigh, as estimated by Finkbeiner for the southern $\mathrm{H} \alpha$ data. For the dust template, the $94 \mathrm{GHz}$ emission predicted by model 8 of Finkbeiner et al. (1999) was used. The adopted zero-level uncertainty is $\pm 0.2 \mu \mathrm{K}$, propagated from a zero-level uncertainty of $\pm 0.044 \mathrm{MJy} \mathrm{sr}^{-1}$ for the $100 \mu \mathrm{m}$ dust map of Schlegel et al. (1998).

Fits were done to $N_{\text {side }}=512$ pixels that are outside of the combined KQ85 plus point-source mask and have optical depth at $\mathrm{H} \alpha$ less than 0.5, based on the Schlegel et al. (1998) extinction map. This pixel selection covers $74 \%$ of the sky. The offsets from the fits are $-25 \pm 19,-5.4 \pm 6.8,-2.2 \pm 3.9$, $-2.2 \pm 1.5$, and $-1.5 \pm 0.7 \mu \mathrm{K}$ in $\mathrm{K}, \mathrm{Ka}, \mathrm{Q}, \mathrm{V}$, and $\mathrm{W}$ bands, respectively. Thus, there is no evidence for significant error in the 5 year map zero levels as determined from the csc $|b|$ fitting. For comparison, Northern Hemisphere csc $|b|$ fits can be used to estimate uncertainties in the zero levels; the Northern 
Hemisphere gives offsets of $-9.2,3.2,3.5,-2.5$, and $-5.9 \mu \mathrm{K}$ for $\mathrm{K}, \mathrm{Ka}, \mathrm{Q}, \mathrm{V}$, and $\mathrm{W}$ bands, respectively, relative to the zero levels calculated from the Southern Hemisphere.

\subsection{Template Cleaning}

The foreground template subtraction technique used in the 5 year analysis is unchanged from that used in the 3 year release. The method was described in Hinshaw et al. (2007) for temperature cleaning and Page et al. (2007) for polarization cleaning; details are not repeated here.

In summary, for temperature cleaning, a model of the foreground emission is computed from a simultaneous fit to the 5 year Q-, V- and W-band maps, and this model is then used to produce foreground-reduced maps suitable for cosmological studies. WMAP has two differencing assemblies (DAs) for $\mathrm{Q}$ and V bands (labeled Q1, Q2, V1, and V2) and four for the $W$ band (labeled W1 through W4), for a total of eight maps with independent noise properties.

The model takes the form

$$
\begin{aligned}
& M(v, p)=b_{1}(v)\left(T_{K}(p)-T_{K a}(p)\right) \\
& +b_{2}(v) I_{\mathrm{H} \alpha}(p)+b_{3}(v) M_{\mathrm{dust}}(p),
\end{aligned}
$$

where $p$ indicates the pixel, the frequency dependence is entirely contained in the coefficients $b_{i}$, and the spatial templates are the WMAP $\mathrm{K}-\mathrm{Ka}$ temperature difference map $\left(T_{K}-T_{K a}\right)$, the Finkbeiner (2003) composite $\mathrm{H} \alpha$ map with an extinction correction applied $\left(I_{\mathrm{H} \alpha}\right)$, and the Finkbeiner et al. (1999) dust model evaluated at $94 \mathrm{GHz}\left(M_{\text {dust }}\right)$. All of these spatial templates are available on LAMBDA.

The $\mathrm{H} \alpha$ map and dust template are based on external data and have not changed since the 3 year analysis. The first template, however, has slightly changed (at the $\sim 10 \mu \mathrm{K}$ level) due primarily to changes in the gain calibration since the 3 year release; see Figure 5 of Hinshaw et al. (2009) for details. Because this template has contributions from both synchrotron and free-free emission, foreground parameters are a mixture of $b_{1}(v)$ and $b_{2}(v)$. For free-free emission, the ratio of the $K$ band radio temperature to $\mathrm{H} \alpha$ intensity is

$$
h_{\mathrm{ff}}=\frac{b_{2}(v)}{S_{\mathrm{ff}}(v)-0.552 b_{1}(v)},
$$

where $S_{\mathrm{ff}}(v)$ is the free-free emission spectrum converted to thermodynamic temperature units and is assumed to be a power law with $\beta=-2.14$. The synchrotron spectral index (relative to the $\mathrm{K}$ band) is found via

$$
\beta_{s}=\frac{\log \left[0.67 b_{1}(v) a(v)\right]}{\log \left(v / v_{K}\right)},
$$

where $a(v)$ is the conversion factor from the antenna temperature to thermodynamic units.

The coefficients of the model fit to the 5 year data are presented in Table 2. Small changes in the 5 year coefficients compared to the 3 year values (Table 5 of Hinshaw et al. 2007) reflect the 5 year updates to absolute calibration and beam profiles. The new template maps are shown in Figure 4.

For polarization cleaning, the maps are degraded to low resolution $\left(N_{\text {side }}=16\right)$. The model has the form

$$
\begin{aligned}
& {[Q(v, p), U(v, p)]_{\text {model }}=a_{1}(v)} \\
& \quad \times[Q(p), U(p)]_{K}+a_{2}(v)[Q(p), U(p)]_{\text {dust }} .
\end{aligned}
$$
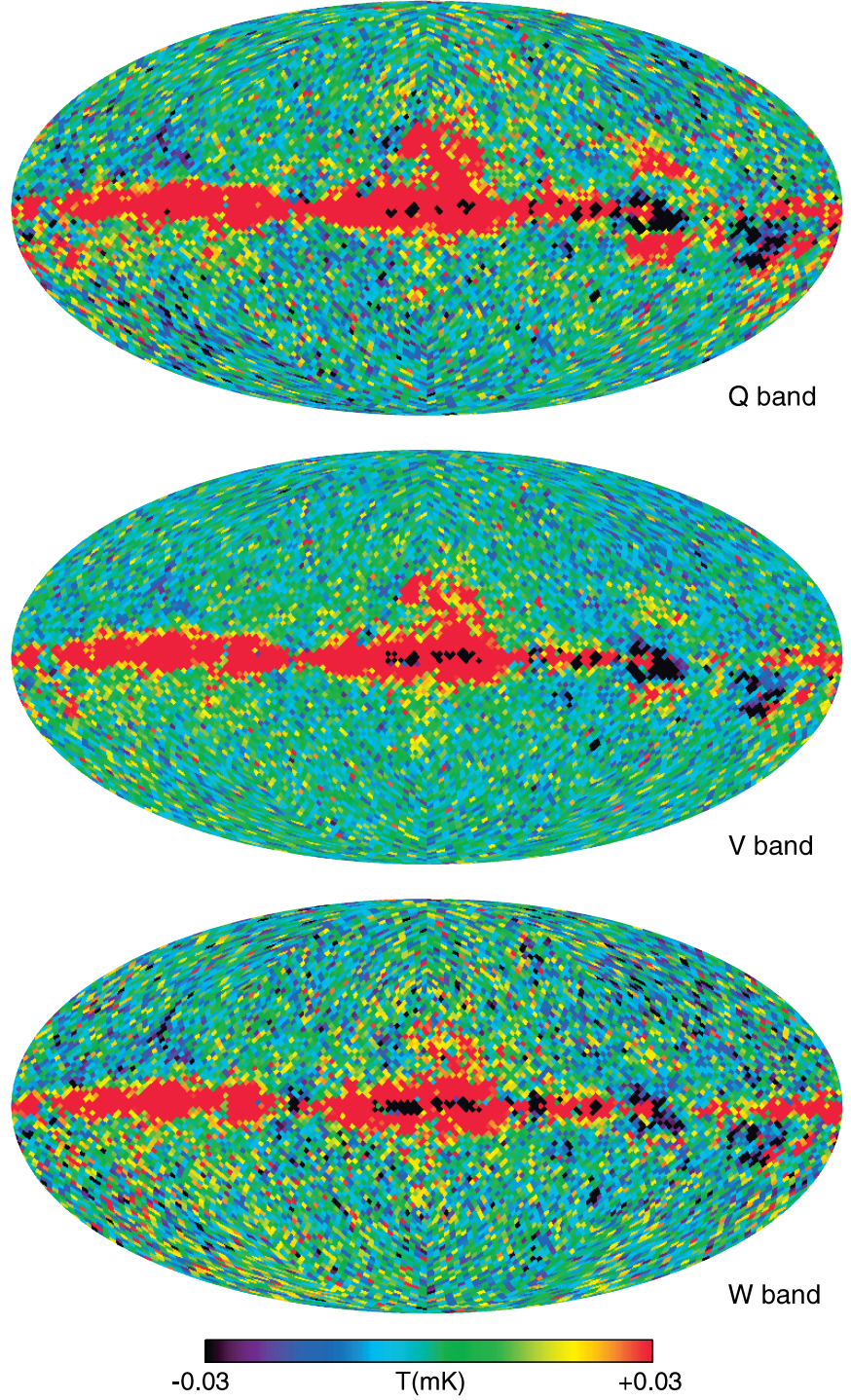

Figure 4. 5 year temperature maps with foregrounds reduced via template cleaning. All maps have had the 5 year ILC estimate for the CMB subtracted, and have been degraded to $N_{\text {side }}=32$. Frequency bands shown are Q, V, and W. Compare to Figure 10 of Hinshaw et al. (2007). Outside the Galactic mask, the template cleaning reduces foregrounds to $\sim 15 \mu \mathrm{K}$ or less.

Table 2

Template Cleaning Temperature Coefficients

\begin{tabular}{lccccc}
\hline \hline $\mathrm{DA}^{\mathrm{a}}$ & $b_{1}$ & $b_{2}\left(\mu \mathrm{K} \mathrm{R}^{-1}\right)$ & $b_{3}$ & $\beta_{s}{ }^{\mathrm{b}}$ & $h_{\mathrm{ff}}{ }^{\mathrm{c}}\left(\mu \mathrm{K} \mathrm{R}^{-1}\right)$ \\
\hline Q1 & 0.245 & 0.981 & 0.201 & -3.18 & 5.99 \\
Q2 & 0.243 & 1.009 & 0.199 & -3.22 & 6.01 \\
$\mathrm{~V} 1$ & 0.058 & 0.666 & 0.461 & -3.44 & 6.38 \\
$\mathrm{~V} 2$ & 0.056 & 0.647 & 0.477 & -3.43 & 6.38 \\
$\mathrm{~W} 1$ & 0.000 & 0.398 & 1.262 & $\cdots$ & 6.62 \\
$\mathrm{~W} 2$ & 0.000 & 0.393 & 1.277 & $\cdots$ & 6.62 \\
$\mathrm{~W} 3$ & 0.001 & 0.398 & 1.242 & $\cdots$ & 6.61 \\
$\mathrm{~W} 4$ & 0.000 & 0.395 & 1.271 & $\cdots$ & 6.62 \\
\hline
\end{tabular}

Notes.

${ }^{a}$ WMAP has two DAs for $\mathrm{Q}$ and $\mathrm{V}$ bands and four for the $\mathrm{W}$ band; the high $\mathrm{S} / \mathrm{N}$ in total intensity allows each DA to be fitted independently.

${ }^{\mathrm{b}}$ Power-law slope relative to the $\mathrm{K}$ band, as derived from $b_{1}$; W band values are less than -4 .

${ }^{\mathrm{c}}$ Free-free to the $\mathrm{H} \alpha$ ratio at the $\mathrm{K}$ band, as derived from $b_{1}$ and $b_{2}$. The expected value for an electron temperature of $8000 \mathrm{~K}$ is $11.4 \mu \mathrm{K} \mathrm{R}^{-1}$ (Bennett et al. 2003). 
The templates used are the WMAP K-band polarization for synchrotron $\left([Q, U]_{K}\right)$, and a low-resolution version of the dust template used above with the polarization direction derived from starlight measurements $\left([Q, U]_{\text {dust }}\right)$. While the dust polarization template maps are unchanged since the 3 year release, further WMAP observations have improved the $\mathrm{S} / \mathrm{N}$ for synchrotron polarization template maps. The coefficients of the model fit to the 5 year data are in Table 3. For polarization, the template maps are assumed to have a one-to-one correspondence to foreground emission, so the spectral indices for synchrotron and dust are simply the power-law slopes of the coefficients $a_{1}(v)$ and $a_{2}(v)$. As was the case for the 3 year data, a fit fixing the synchrotron spectral index was found to have no influence on cosmological conclusions and was not used for analysis.

\section{MCMC FITTING}

\subsection{Description}

The analysis is carried out with band-averaged maps at each frequency, which are calibrated in antenna temperature, smoothed to a $1^{\circ}$ Gaussian beam, and pixelized using an $N_{\text {side }}=64$ HEALPix grid. This makes the fit computationally manageable and ensures that pixel-pixel correlations are small, simplifying the error description. The maps use the csc $|b|$ fit process described above to determine the zero point.

Next, we parameterize the emission in each pixel with a physical model. The model depends on the parameters in a nonlinear way and the parameters can be highly correlated. A Monte Carlo chain is run for each pixel to determine the probability distribution for the parameters of the model using the Markov chain technique (Gilks et al. 1996). Because of parameter correlations, the matrix describing the optimal step size is not diagonal. The starting points and initial step proposal matrices are generated using a "best guess" from the data. In cases where the initial guess turns out to be poor, the fitting process is retried using the existing chain to improve the guess. Any retries or poorly conditioned proposal matrices are flagged. Each chain is checked for convergence using the criteria described in Dunkley et al. (2005), and any lack of convergence is also flagged.

The basic form of the model for each pixel is

$T(v)=T_{s}\left(\frac{v}{v_{K}}\right)^{\beta_{s}(v)}+T_{f}\left(\frac{v}{v_{K}}\right)^{\beta_{f}}+a(v) T_{\mathrm{CMB}}+T_{d}\left(\frac{v}{v_{W}}\right)^{\beta_{d}}$

for the antenna temperature and

$$
\begin{aligned}
& Q(v)=Q_{s}\left(\frac{v}{v_{K}}\right)^{\beta_{s}(v)}+Q_{d}\left(\frac{v}{v_{W}}\right)^{\beta_{d}}+a(v) Q_{\mathrm{CMB}} \\
& U(v)=U_{s}\left(\frac{v}{v_{K}}\right)^{\beta_{s}(v)}+U_{d}\left(\frac{v}{v_{W}}\right)^{\beta_{d}}+a(v) U_{\mathrm{CMB}}
\end{aligned}
$$

for Stokes $Q$ and $U$ parameters. The subscripts $s, f, d$ stand for synchrotron, free-free, and dust emission, $v_{K}$ and $v_{W}$ are the effective frequencies for $K$ and $W$ bands $(22.5$ and $93.5 \mathrm{GHz})$, and $a(v)$ accounts for the slight frequency dependence of a $2.725 \mathrm{~K}$ blackbody, respectively, using the thermodynamic to antenna temperature conversion factors found in Bennett et al. (2003).

For each pixel, the $\chi^{2}$ of the fit is then calculated in the standard way

$$
\chi^{2}=\sum_{v} \mathbf{D}_{v}^{T} \mathbf{N}_{v}^{-1} \mathbf{D}_{v}
$$

Table 3

Template Cleaning Polarization Coefficients

\begin{tabular}{lcccc}
\hline \hline Band & $a_{1}{ }^{\mathrm{a}}$ & $\beta_{s}\left(v_{K}, v\right)^{\mathrm{b}}$ & $a_{2}{ }^{\mathrm{a}}$ & $\beta_{d}\left(v, v_{W}\right) \mathrm{b}$ \\
\hline$K a$ & 0.3161 & -3.17 & 0.0165 & 1.35 \\
$Q$ & 0.1765 & -3.04 & 0.0147 & 1.85 \\
$V$ & 0.0595 & -2.96 & 0.0366 & 1.58 \\
$W$ & 0.0450 & -2.35 & 0.0822 & $\cdots$ \\
\hline
\end{tabular}

Notes.

${ }^{\text {a }}$ The $a_{i}$ coefficients are dimensionless and produce model maps from templates.

$\mathrm{b}$ The spectral indices refer to antenna temperature.

where $\mathbf{D}_{v}$ is the difference between the data vector $(T, Q, U)$ and the model vector at each frequency. The matrix $\mathbf{N}_{v}$ is the noise covariance matrix, and is directly derived from the $N_{\text {obs }}$ maps (rebinned from an $N_{\text {side }}$ of 512 to an $N_{\text {side }}$ of 64), with minor modifications discussed below.

Not all parameters in the model are free to vary; $a(v)$ and $\beta_{f}$ are fixed by known physics, the $Q$ and $U$ parameters for foregrounds are related by using the $K$ band as a template for the polarization angle, and, for most of the following, $\beta_{s}(v)$ is assumed to be constant with frequency (though allowed to vary spatially). The free-free index was fixed at $\beta_{f}=-2.14$; the typical variation in this value at $W M A P$ frequencies is \pm 0.015 (Oster 1961, also see Quireza et al. 2006; Itoh et al. 2000 for recent refinements), which is too small for WMAP to detect. Similarly, results of the fit were not found to strongly depend on whether $U_{s}$ and $U_{d}$ were treated as freely independent parameters.

The "base" fit, which allows for spatially varying synchrotron and dust spectral indices, has 10 independent parameters per pixel: $T_{s}, T_{f}, T_{d}, T_{\mathrm{CMB}}, \beta_{s}, \beta_{d}, Q_{s}, Q_{d}, Q_{\mathrm{CMB}}$, and $U_{\mathrm{CMB}}$. More restricted fits fixed $\beta_{s}, \beta_{d}$, or both. Other fits allowed for a frequency-dependent $\beta_{s}$ by defining

$$
\beta_{s}(v)=\beta_{s}+c \ln \left(v / v_{K}\right),
$$

where the new parameter $c$ can introduce a gradual steepening (or shallowing).

Note that the models used here assume that polarized and unpolarized synchrotron emission have the same spectral behavior. While this assumption appears to be safe at high latitudes, it may not be accurate for LOS that pass through the Galactic plane. This is further explored in Section 5.

Finally, some fits allowed for an additional independent component, either using the exact "cold neutral medium" (CNM) spectrum for spinning dust (Draine \& Lazarian 1998), or using a generalized analytic form

$$
T_{s d}(v)=A_{s d} \frac{\left(v / v_{s d}\right)^{\beta_{d}+1}}{\exp \left(v / v_{s d}\right)-1}
$$

The analytic form is a modified blackbody with the amplitude, low-frequency spectral index, and turnover frequency explicitly decoupled from one another. In Figure 5, plots of both the exact and "shifted" spectra used in the fitting process are shown, as well as a curve showing that the analytic form is indeed a good approximation to the numerically calculated spectrum. In practice, the low-frequency spectral index is irrelevant because the desired shape for foreground fitting has $v_{s d}$ well below $22 \mathrm{GHz}$ and is thus dominated by the exponential cutoff. While this form was motivated as an analytic approximation to spinning dust spectra, it could also represent a variety of 


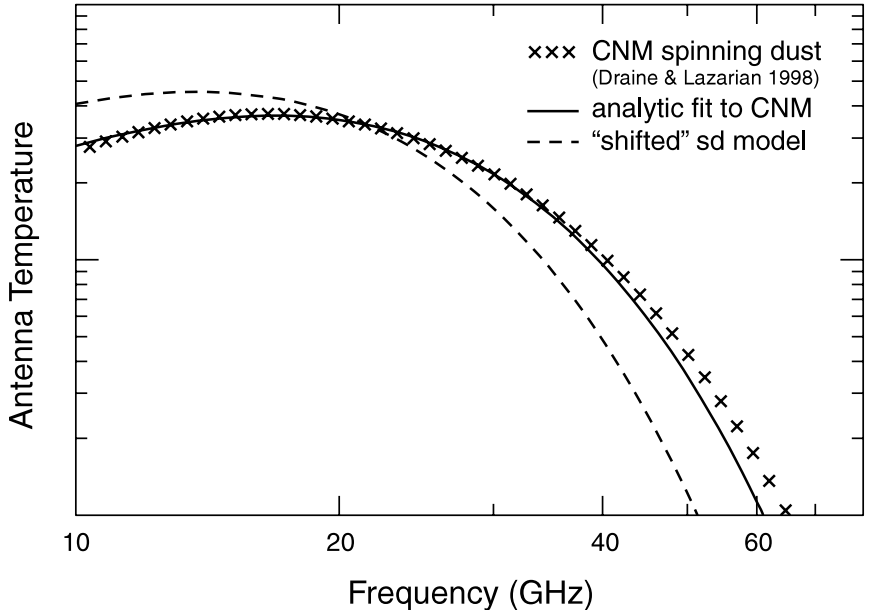

Figure 5. Exact CNM spinning dust spectrum as calculated by Draine \& Lazarian (1998), an analytical fit to the model, and the ad hoc "shifted" model, which better fits radio observations in the Galactic plane. The shifted model may represent a mix of warm neutral medium and warm ionized medium models, or another emission process entirely. The vertical axis is in units of antenna temperature, but the overall scale is arbitrary. Agreement between the exact model and the analytic approximation is better than $5 \%$ over the frequency range from 15 to $35 \mathrm{GHz}$. This is smaller than the fractional error from fits that include a spinning dust component.

other physical sources of microwave emission. This component was assumed to have no significant polarization.

Each chain itself is a multistep process. The code makes an initial guess for the best-fit parameters and runs for a burn-in period to find the region of parameter space near minimum $\chi^{2}$. There is then a "prechain" to find the approximate moments of the likelihood; these moments are used to optimize the proposal distribution for the final chain. Problems at any stage due to lack of convergence or poorly characterized parameter distributions are flagged and recorded; only rarely are more than $0.5 \%$ of pixels so affected, and most problems are due to random fluctuations and disappear with longer chains.

\subsection{Tests and Sources of Error}

The Monte Carlo process (with Metropolis steps) has the advantage that it can sample the full parameter space and will converge on the likelihood even if the likelihood is non-Gaussian or unknown a priori. The disadvantage is that degeneracies in parameter space will slow the convergence, and cutting off regions of parameter space to improve convergence can bias the results. The prime example of this is degeneracy between synchrotron and free-free emission amplitude. If the synchrotron spectral index is allowed to flatten to the freefree value, then the amplitudes of the two components become degenerate parameters, which can distort the fit.

We test this with simulated maps where the input foreground is known. To ensure that the noise properties of the maps are well understood, we used extensive simulations. We combined the high-resolution noise information with the low-resolution pixel-pixel covariance in order to generate noise realizations that are as realistic as possible, which are then smoothed by using the same process used for the real sky maps. We then produce mock sky maps with CMB realizations synthesized from the WMAP "best-fit" $\Lambda$ cold dark matter $(\Lambda \mathrm{CDM})$ model, noise realizations from the 5 year noise covariance matrix, and foregrounds generated from a variety of models.
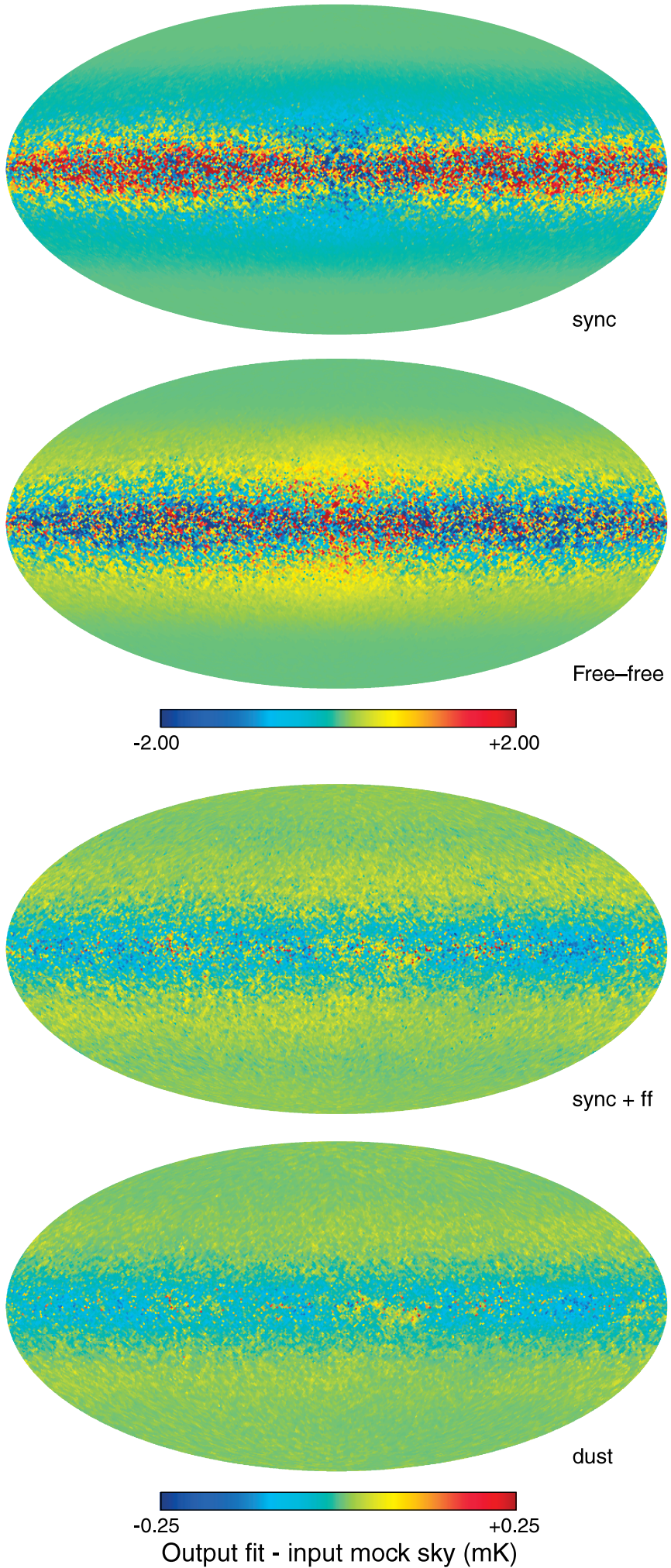

Figure 6. Difference maps between input and output foregrounds for the mock sky reconstruction. For comparison, the peak temperature for synchrotron plus free-free inputs was $17 \mathrm{mK}$, and for the input dust, the peak was $1.9 \mathrm{mK}$. The degeneracy between synchrotron and free-free emission means that their sum is much better constrained than either component individually. The scatter is larger than the bias, which is small compared to the input signal and within the error estimate given by the fit. These degeneracy issues are also illustrated in Figure 7. Synchrotron and free-free antenna temperatures are defined as measured at the $\mathrm{K}$ band and dust as measured at the $\mathrm{W}$ band. 


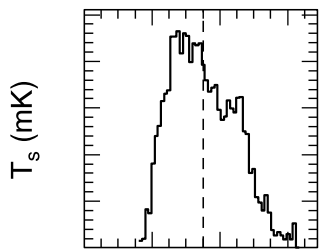

Histograms for one pixel of mock sky at galactic coordinates $33.7+30.7$
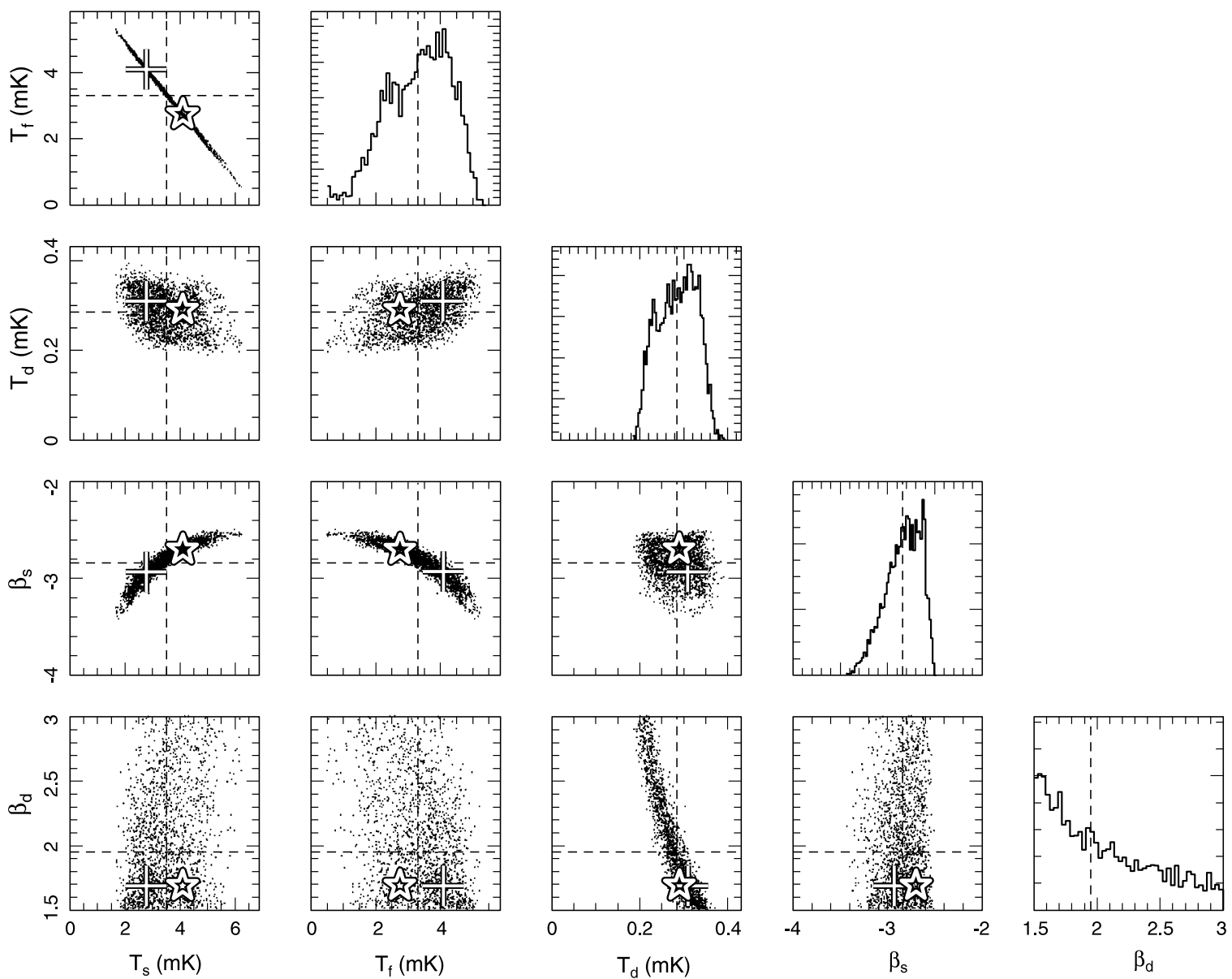

Figure 7. Histograms for foreground parameters for a single pixel of mock sky reconstruction. The mock foregrounds were designed to have the same statistical behavior as the true sky but do not match in detail. The same pixel is examined for the true sky in Figure 8. Dashed lines indicate the mean of the chain, crosses indicate the best-fit point, and stars indicate the input values for the parameters. The strong $T_{s}-T_{f}$ degeneracy and the curved $T_{s}-\beta_{s}$ degeneracy are typical for high $\mathrm{S} / \mathrm{N}$ pixels. Other parameter correlations are minor. Both the mean and best-fit parameters of the chain are within the expected error range from the input values.

When degeneracies exist, the random fitting process tends to share the amplitude evenly between degenerate parameters. This can lead to biasing if the true sky also does not have equal contributions from such parameters. This effect can be clearly seen by comparing the reconstruction of the synchrotron and free-free components (Figure 6). Figure 7 shows histograms of a single pixel chain from the mock sky fit, with the input values of the parameters marked with a bold cross.

The fitting process uses the error information contained in the $N_{\text {obs }}$ maps, which includes covariance between the $Q$ and $U$ Stokes parameters within a pixel, but covariance between pixels (due to low-frequency noise or the smoothing process) is not included. The correlation coefficient between adjacent pixels ranges from less than 0.20 up to 0.45 (for the $K$ band and $W$ band, respectively) due to the smoothing process. The fit treats pixels as independent, essentially marginalizing over all other pixels when fitting; so the main effect of the correlations is to introduce similar small pixel-pixel correlations in the $\chi^{2}$ values.
This has a negligible effect on the results as long as goodness of fit is averaged over large enough regions.

Smoothing also reduces the overall noise level, and this has been modeled through direct noise simulations and accounted for in the fit process. The method used was to generate many realizations of simulated noise maps based on the original $N_{\mathrm{obs}}$ information, smooth them using the same window functions used to smooth the real data, and fit a Gaussian shape to a histogram of the result. From this process, an overall multiplicative rescaling factor was determined for each frequency band and applied to the $N_{\text {obs }}$ files used for the final fit. With this correction, the $\chi^{2}$ per degree of freedom should be close to unity for an ideal fit; for the mock fit described above, the mean (per pixel) reduced $\chi^{2}$ was 1.11 with 7.2 degrees of freedom (DOF).

Small differences in the beam solid angle from one frequency band to another can distort the inferred spectral index, especially near bright sources. We used the Jupiter-based beam maps from Hill et al. (2009) and smoothed them to a common $1^{\circ}$ beam, 


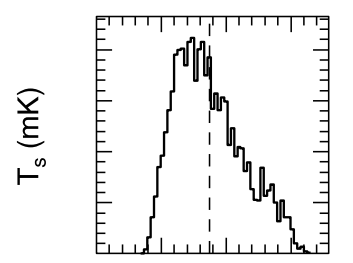

Histograms for one pixel of observed sky at galactic coordinates $33.7+30.7$
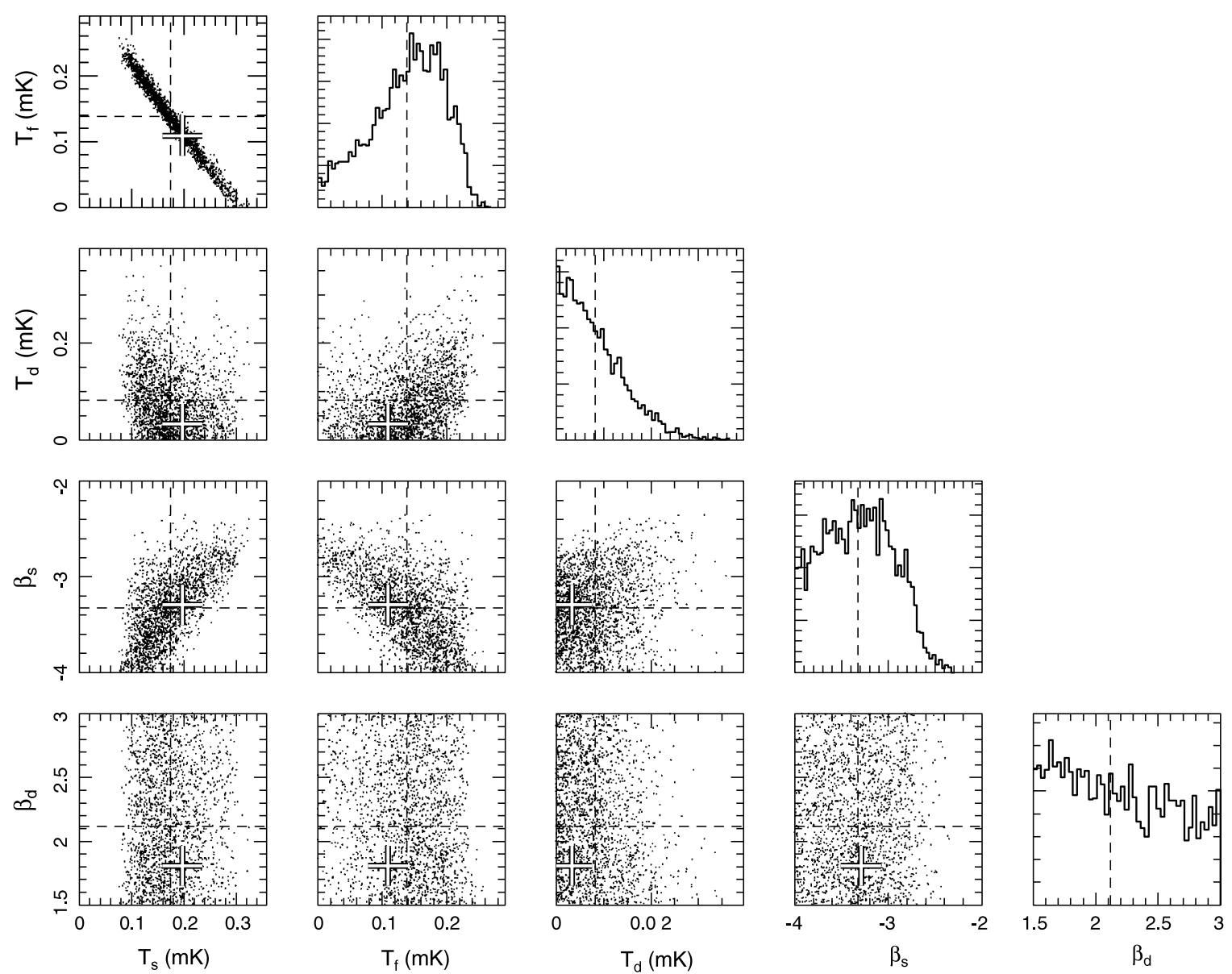

Figure 8. Histograms for foreground parameters for a single pixel of the observed sky from actual WMAP data, and not simulation. Dashed lines indicate the mean of the chain and crosses indicate the best-fit point. The observed sky shows the same basic behavior as the simulated sky used for testing.

similar to how the sky maps are smoothed, and found that beam systematics at the known level can cause deviations of up to \pm 0.1 in the spectral index. Errors of this type (that are multiplied by the sky signal) are included in the fit by adding $0.3 \%$ of the antenna temperature to the error budget for each pixel. This number was derived from the observed scatter one beam-width away from bright point sources. Systematics of this type do not average down, and so can quickly become dominant at low resolutions.

The smoothing kernel used to match the bands to $1^{\circ}$ resolution uses the symmetrized beam profile, and hence does not take into account beam asymmetries. WMAP's observational strategy, however, symmetrizes the beam to a large extent. Page et al. (2003) investigated the extent to which remaining beam asymmetry could affect the beam window functions and found it to be $<1 \%$. Any effect on the maps due to beam asymmetries should be weak near the ecliptic poles and for extended emission not aligned along the plane of the ecliptic.

There remain small uncertainties of a few $\mu \mathrm{K}$, both in the true zero level of the maps and in the dipole subtraction process. The offsets primarily affect foreground estimation by changing the apparent spectral index when averaging over large, very low $\mathrm{S} / \mathrm{N}$ regions. We avoid this by explicitly deweighting or masking pixels with weak spectral index constraints when reporting results. Second, as a purely pixel-based method, the MCMC foreground fitting process we use is free to produce foreground (or even $\mathrm{CMB}$ ) maps with nonzero monopole and dipole contributions. The sensitivity of the fit to offsets was checked by adding offsets of $100 \mu \mathrm{K}$ (several times larger than the error as estimated from the csc $|b|$ fits) to the sky maps and repeating the analysis. No foreground component was found to change by more than $10 \%$ for pixels where the $\mathrm{S} / \mathrm{N}$ was significant.

\section{FIT RESULTS AND COMPARISONS}

\subsection{MCMC Fit}

Each pixel fit consists of 15 data points (Stokes $I, Q$, and $U$ for each of the five frequency bands), and a foreground fitting model can use from 8 to 12 parameters per pixel. The fitting process produces a $\chi^{2}$ value for each pixel. Normally, the reduced $\chi^{2}$ is found by dividing by the number of DOF. However, the true 
Table 4

Model Fits to WMAP Temperature and Polarization Data

\begin{tabular}{|c|c|c|c|c|}
\hline \multirow[t]{2}{*}{ Model } & \multirow{2}{*}{$\begin{array}{c}\text { \# of } \\
\text { Params }\end{array}$} & \multicolumn{3}{|c|}{ Best-Fit $\chi_{v}^{2}$ a } \\
\hline & & Outside Plane $^{\mathrm{b}}$ & Inside Plane $^{\mathrm{b}}$ & Full Sky \\
\hline Base & 10 & 1.14 & 2.23 & 1.24 \\
\hline Base + Haslam & 10 & 1.14 & 2.36 & 1.26 \\
\hline Loose priors & 8 & 1.09 & 3.26 & 1.29 \\
\hline Steep & 10 & 1.14 & 0.97 & 1.13 \\
\hline Exact sd & 9 & 1.21 & 1.63 & 1.25 \\
\hline Shifted sd & 9 & 1.24 & 1.00 & 1.22 \\
\hline$\beta_{s}=-3.2, \beta_{d}=1.7$ & 8 & 1.16 & 4.33 & 1.45 \\
\hline$\beta_{s}=-2.6, \beta_{d}=1.7$ & 8 & 1.30 & 3.42 & 1.50 \\
\hline$\beta_{s}$ variable, $\beta_{d}=1.7$ & 9 & 1.16 & 2.92 & 1.32 \\
\hline$\beta_{s}$ variable, $\beta_{d}$ variable & 10 & 1.14 & 2.23 & 1.24 \\
\hline
\end{tabular}

Notes.

a Reduced $\chi^{2}$ averaged over pixels in the region, with effective degrees of freedom determined by the chain. The statistical errors are less than 0.01 .

$\mathrm{b}$ The mask used to define these regions is a smoothed version of the $95 \%$ mask, the 5 year release analogue of the Kp12 mask.

number of DOF in this case is difficult to determine because neither the data points nor the fitting parameters are statistically independent of one another. Using the MCMC chain for each pixel, though, it is possible to use the "Bayesian complexity" (described in a cosmological context in Kunz et al. 2006), defined as the difference between the average $\chi^{2}$ over the chain and the $\chi^{2}$ of the best fit. This serves as a measure of the effective number of DOF, and can then be used to determine the reduced $\chi^{2}$ per pixel. Using the simulated skymaps described above, we have found that the statistical behavior of the reduced $\chi^{2}$ defined this way is consistent with that of a $\chi^{2}$ distribution. This "effective" reduced $\chi^{2}$ is how we quantify the goodness of fit in the tables and figures.

Pixels with high reduced $\chi^{2}$ are not being well fit by the model. Since such pixels are largely confined to the plane, the sky was divided into regions "outside" and "inside" the Galactic plane by using progressively smaller masks until the average "outside" $\chi^{2}$ was no longer independent of the mask. Regions near known point sources from Wright et al. (2009) are excluded from all analysis, both inside and outside of the plane, leaving $92 \%$ of the full sky. Flagged pixels are also not included; for most fits, such pixels arise from poorly conditioned covariance matrices, are uniformly distributed, and make up less than $0.5 \%$ of the sky.

The $\chi_{v}^{2}$ results of several fits are shown in Table 4 . Histograms for a single pixel chain are shown in Figure 8 . The mock sky simulations appear to capture the basic behavior of the parameter correlations. Detailed comparisons of specific results are given in the following subsections. Figure 9 shows the basic results and Figure 10 shows the temperature residuals of the best-fit base model subtracted from the data, in units of $1 \sigma$ of noise. Figure 11 shows the difference between the MCMC fit and the 5 year MEM fit. The overall fit outside the complex and troublesome Galactic plane region approaches a $\chi_{v}^{2}$ of 1.14 and the residuals are mostly randomly distributed, which suggests that the overall fit works reasonably well and that the noise properties have been properly described.

The "base" model uses the 10 parameters described above. Another fit is done including data from $408 \mathrm{MHz}$ (Haslam et al. 1981), assuming $10 \%$ calibration errors. As a check, a "loose priors" fit is done, which allows foreground temperatures to become negative. For this fit to converge, the spectral indices
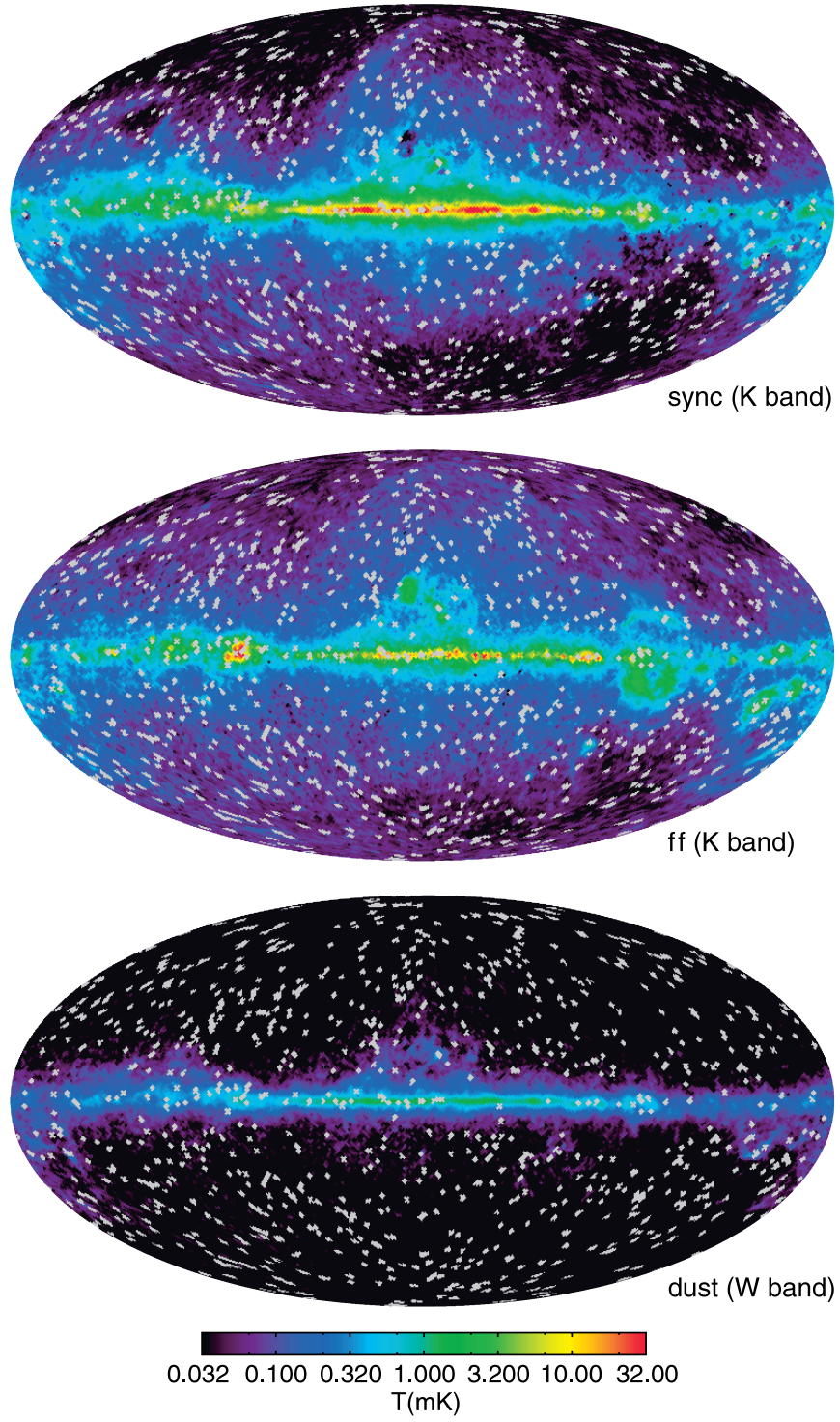

Figure 9. Temperature maps for foreground components as determined by the MCMC fitting process for the "base" model. Maps from other models are qualitatively similar. Synchrotron and free-free temperatures are as measured at the $K$ band; dust is measured at the $W$ band. Gray pixels are those masked due to point sources or flagged as problematic. Top: synchrotron, middle: free-free, bottom: dust.

must be fixed, however, so it only uses eight parameters. The "steep" model fixes the dust spectral index but allows for a synchrotron steepening parameter $c$, as described above. The "exact sd" model uses the CNM spinning dust spectrum for an additional foreground, whereas the "shifted sd" model uses the generalized spectrum with $v_{\mathrm{sd}}=4.9 \mathrm{GHz}$. In both cases, the synchrotron and dust spectral indices are fixed to be the same at each pixel (again for convergence reasons). Finally, several fits were done with fixed spectral indices to examine the effect of using different values, shown as the last part of Table 4. The last entry of the table is the "base" model, repeated for ease of comparison.

\subsection{Overall Foreground Features}

Figures 9-14 show maps of the results from the "base" fit. Figures 9 and 12 show the three foregrounds themselves and their errorbars as determined from the parameter variance in the Markov chains. The maps are in units of antenna temperature as 

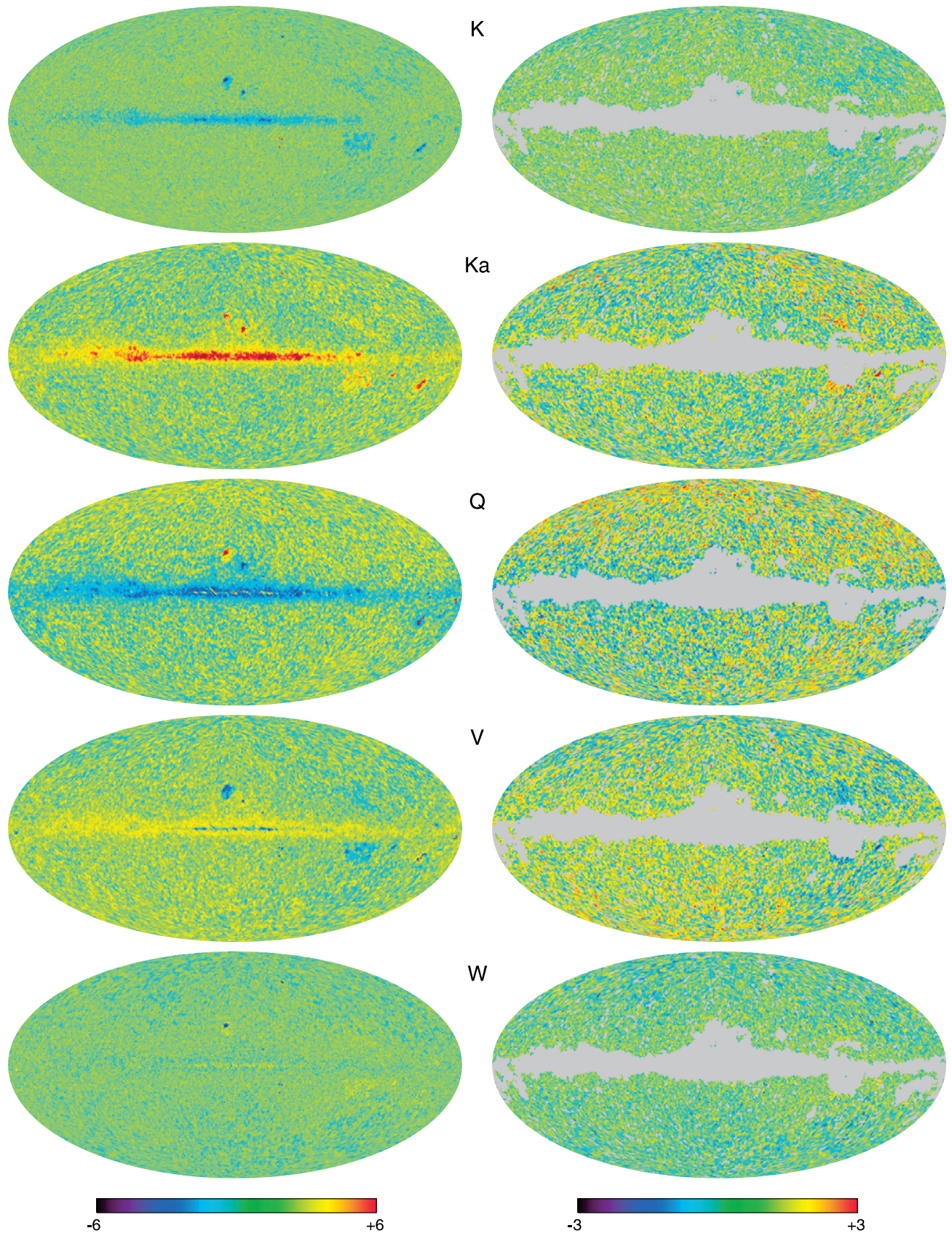

Figure 10. Residuals of the "base" model foreground fit subtracted from the data. The left column shows the residuals in units of noise sigma, from -6 to 6 . The right column has the $85 \%$ mask applied and a scale of -3 to 3 . Frequency bands are (from top to bottom) $\mathrm{K}, \mathrm{Ka}, \mathrm{Q}, \mathrm{V}, \mathrm{W}$. The main feature is that the model underestimates Galactic flux in the Ka band and overestimates it in the Q band by a factor of 3-5 times the pixel noise. Outside the analysis mask, the residuals to the model are consistent with noise at the expected level.

measured at the $\mathrm{K}$ band for synchrotron and free-free emission, and at the $W$ band for dust emission. Figure 13 shows spectral index maps binned to lower resolution, where color indicates the spectral index and the size of the circle indicates the significance of the fit result at that location. Figure 14 shows the best $\chi_{v}^{2}$ value achieved at each pixel on the sky.

Almost regardless of foreground model the fit works quite well outside the Galactic plane, giving low $\chi_{v}^{2}$ and foreground maps that are in good agreement with the MEM templates (Hinshaw et al. 2007) and other works (Eriksen et al. 2008). Error maps for synchrotron and free-free emission have similar morphology due to the degeneracy between their amplitudes.

The overall dust brightness seems to be largely consistent with the template prediction (Finkbeiner et al. 1999), though the fit appears to prefer a spatial distribution somewhat less sharply peaked toward the Galactic center (Figure 15). The excess of observed emission compared to that predicted at $90 \mathrm{GHz}$, seen in the original model comparison with Cosmic Background Explorer (COBE) Differential Microwave Radiometer (DMR) data, is still present. Since the fit in the plane has high $\chi^{2}$ and is untrustworthy, the overall preferred spectral index for dust may be $<2.0$, but its significance is not high. Weighting with the covariance information from the fit and masking low-signal regions, the average value for $\beta_{d}$ in the "base" fit is 1.8 with \pm 0.3 from statistical errors and \pm 0.2 from systematic error, depending on how the cuts are defined. For comparison, model 8 of Finkbeiner et al. (1999) predicts $\beta_{d}=1.55 \pm 0.01$ for a comparable sky cut. 

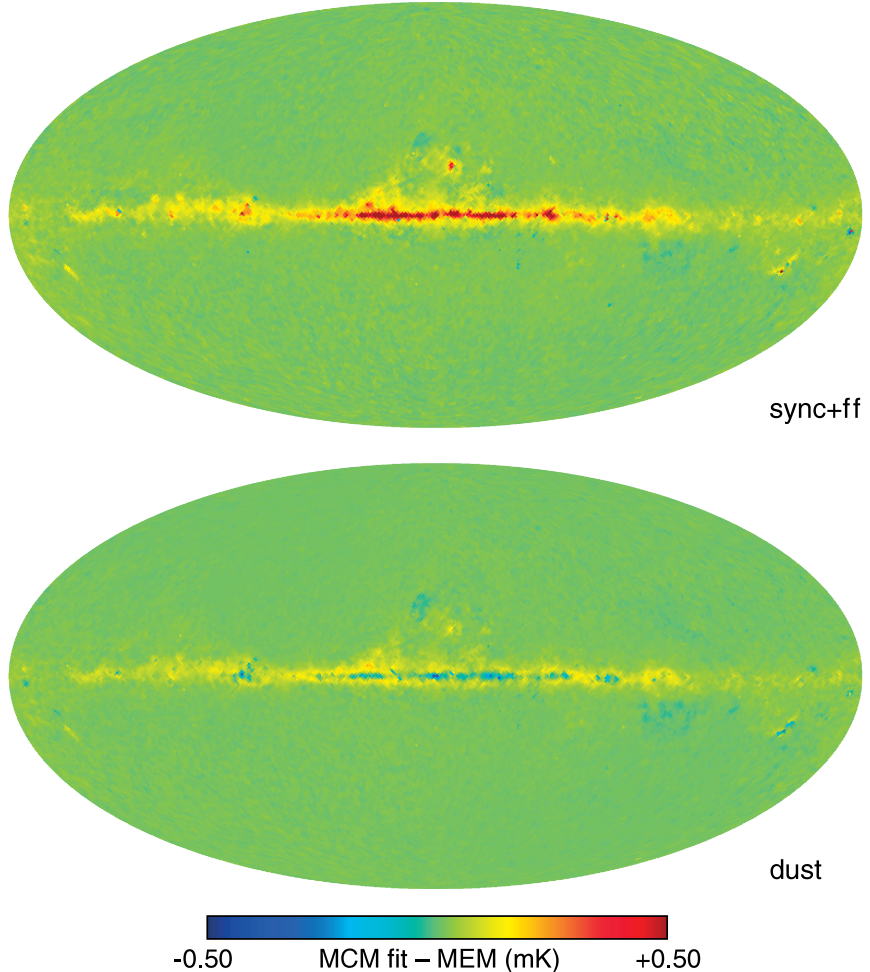

Figure 11. Difference between the MCMC fit and MEM maps. The top panel is the difference (at the K band) in synchrotron plus free-free emission. Differences between these two components separately are larger due to the degeneracy direction. The bottom panel is the difference (at the $\mathrm{W}$ band) in the dust maps. The differences are roughly $1 \%$ of total emission at the $\mathrm{K}$ band and a few percent at the $\mathrm{W}$ band.

The free-free component is consistent with expectations from previous fits and with $\mathrm{H} \alpha$ observations when dust obscuration is taken into account. Free-free emission is quite high in the Galactic plane, and in several regions (including Gum and Orion), it appears to be dominant over synchrotron, even in the $\mathrm{K}$ band. The ratio of the "base" fit free-free map to extinctioncorrected $\mathrm{H} \alpha$ map of Finkbeiner (2003) was used to make a map of $h_{f f}$ (the temperature- $\mathrm{H} \alpha$ intensity conversion factor). A histogram was then made of all pixels with intensities larger than 5 Rayleighs (to mask out low-signal regions) and less than 1 mag of extinction (using the reddening map of Schlegel et al. 1998). A Gaussian fit to the peak of the histogram gives $h_{f f}=11.8 \pm 8.8 \mu \mathrm{K} \mathrm{R}^{-1}$ at the $K$ band, comparable to the value of $11.4 \mu \mathrm{K} \mathrm{R}^{-1}$ expected from an electron temperature of $8000 \mathrm{~K}$ (Bennett et al. 2003), but also consistent with the lower values from template cleaning.

While subject to degeneracy with the free-free emission, synchrotron radiation is a stronger signal in WMAP data than dust emission. Pixel-by-pixel constraints become poor far from the plane; however, there are still constraints on the best-fit spectral index. For example, by comparing fits with a constant spectral index, the Northern Polar Spur and the Fan region prefer an index of -3.0 or steeper. All fits that include total intensity data, show the same preference for a shallower spectral index in the plane as concluded by Bennett et al. (2003); Figure 16 shows $\beta_{s}$ as a function of latitude for a number of different fits.

From the polarized data, the synchrotron polarization fraction indicates strong depolarization toward the Galactic plane consistent with Kogut et al. (2007). Since Faraday rotation should not be large at these frequencies, this effect is due to multiple
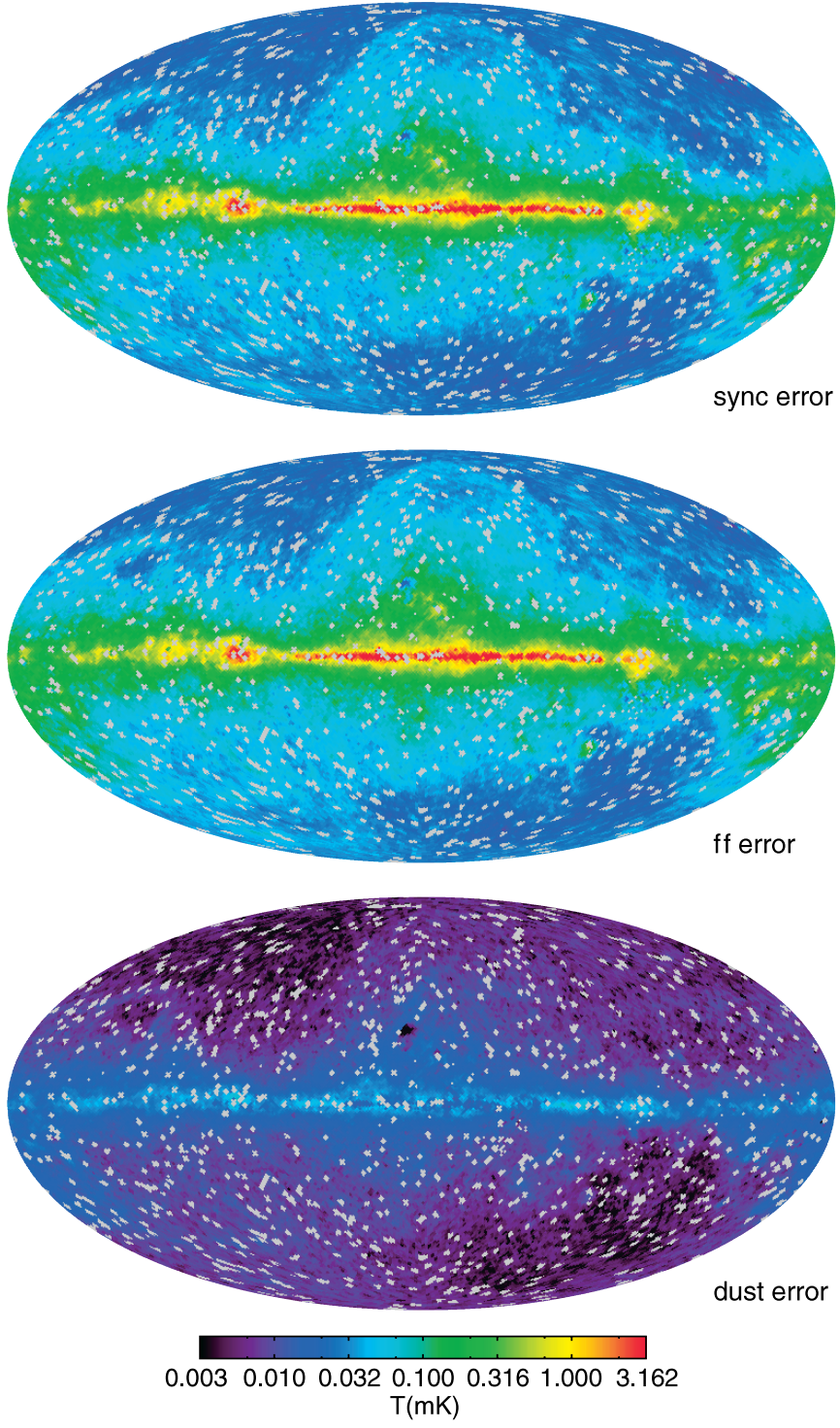

Figure 12. Error maps for foreground components as determined from the marginalized variance given by the MCMC fitting process. Error maps for synchrotron and free-free emission are similar due to the parameter degeneracy between them. Synchrotron and free-free temperatures are as measured at the $\mathrm{K}$ band; dust is measured at the $\mathrm{W}$ band. Gray pixels are those masked due to point sources or flagged as problematic. Top: synchrotron, middle: free-free bottom: dust.

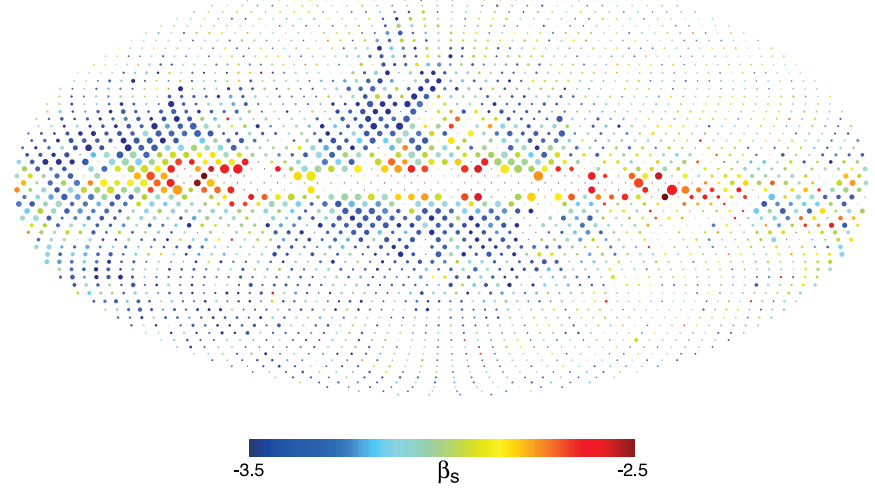

Figure 13. Map of the synchrotron spectral index for the "base" fit, binned to $N_{\text {side }}=16$. Color shows the value of the spectral index and the circle area indicates the weight $\sigma_{\beta}{ }^{-2}$ given by the fit. Pixels with $\chi_{v}^{2}>2$ were explicitly deweighted. 


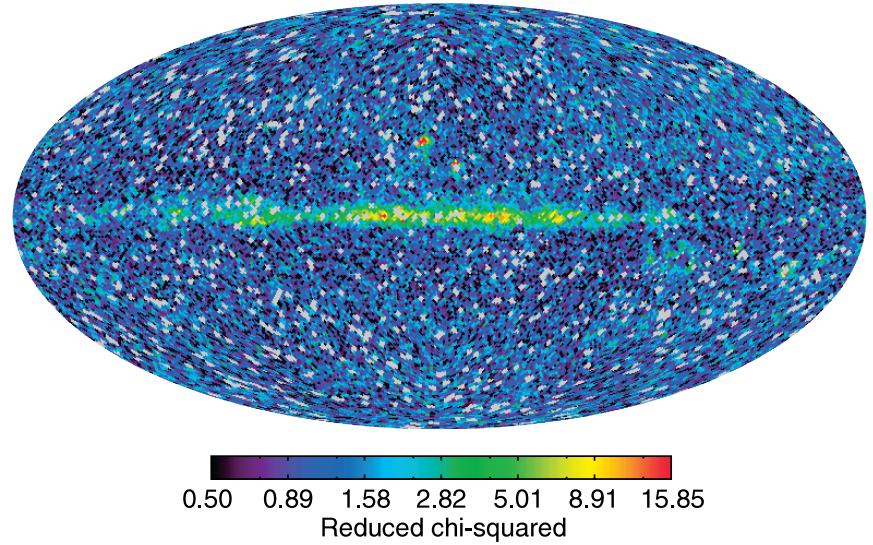

Figure 14. Map of the reduced $\chi^{2}$ per pixel achieved by the "base" fit. Except for a slight excess of high $\chi^{2}$ values from the plane, the overall statistical distribution is that of an ideal $\chi^{2}$ distribution.

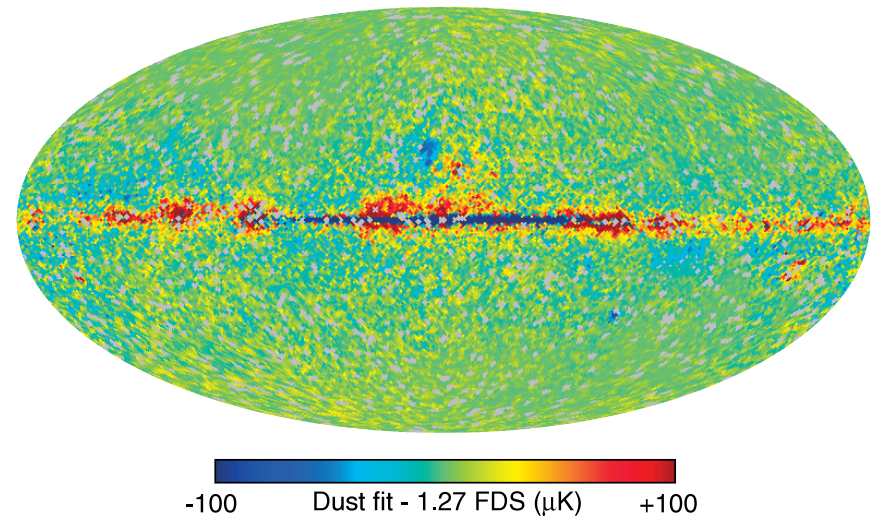

Figure 15. Residuals between the best-fit dust map from the "base" model and 1.27 times the "model 8" dust map of Finkbeiner et al. (1999) extrapolated to $94 \mathrm{GHz}$. Pixels with $\chi_{v}^{2}>5$ or covered by the point-source mask are colored gray. The MCMC fit prefers less dust emission directly toward the Galactic center (deep blue pixels) and somewhat more emission further away (red and orange pixels). Units are antenna temperature at $94 \mathrm{GHz}$.

magnetic field orientations along the line of sight (LOS). Dust polarization fraction appears to follow a pattern similar to the synchrotron polarization fraction, though the $\mathrm{S} / \mathrm{N}$ is low. This is physically reasonable, as the polarization fraction is largely affected by the coherence of the magnetic field along the LOS. This implies that the dust intensity times a constant fraction may not be the best template to use for dust polarization in the Galactic plane.

\subsection{The Galactic Plane}

Regions at very low latitudes are not as well fit by the "base" model, and there is dependence on both foreground model and fit parameters. A map of poorly fit regions reveals that they are in the brightest parts of the Galaxy, where at these frequencies, the free-free emission dominates.

Pixels poorly fit by the "base" model have some common characteristics. Most are bright, but this is probably because similar less bright pixels have lower signal to noise and, thus, lower $\chi^{2}$. Many have a K-Ka temperature spectral index that is similar to what one would expect from free-free emission, but a considerably steeper Ka-Q spectral index. Such pixels tend not to be highly polarized, and the polarized emission that exists appears to be consistent with synchrotron emission

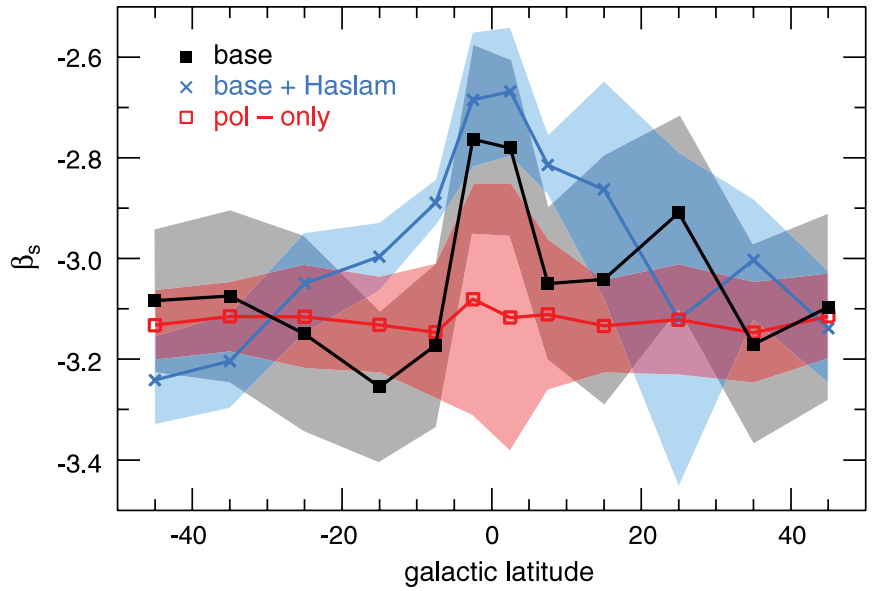

Figure 16. Synchrotron index plotted as a function of latitude for several fits. Pixels are binned by latitude, and only longitudes between $350^{\circ}$ and $10^{\circ}$ are included. Error regions indicate the $68 \%$ scatter within each bin. Solid black squares (gray region) are for the "base" model, blue crosses (blue region) are for the "base" model with $408 \mathrm{MHz}$ data, and red empty squares (red region) are for a fit using only WMAP's polarization data. Fits where the dust spectral index was fixed to 1.7 and 2.0 are almost identical to the "base" fit. The trend to a flatter spectral index in the plane does not appear when only polarization data are used, but the signal to noise is not high enough for the discrepancy to be significant.

with a typical spectral index of $\beta \approx-3$. Data for several such individual pixels are shown in Figure 17.

Figure 18 shows the low-frequency foregrounds given by the MCMC fit using the "steep" model. Thermal dust emission is indistinguishable from the the "base" fit. Residual maps from this fit are featureless, as hinted at from the $\chi^{2}$ information in Table 4. Figure 19 shows low-frequency foregrounds and residuals in $\mathrm{K}, \mathrm{Ka}$, and Q bands for the "shifted sd" fit, which includes a spinning dust-like component. This model produces a good fit in the plane, but seems to have some problems with the Ophiuchus and Gum regions.

For the published CNM spinning dust model (the "exact" model of Table 4), the maximum fraction of the Ka-band flux attributable to spinning dust is $17 \%$ outside of the Galactic plane (using the $85 \%$ mask). The maximum full sky fraction of the Ka-band flux attributable to spinning dust is $20 \%$ for this model. However, this model still does not provide a good fit within the Galactic plane $\left(\chi_{v}^{2}\right.$ in this region is 1.63).

Allowing the spinning dust spectrum to shift in frequency to obtain a better fit results in a Ka-band flux fraction of $14 \%$ for spinning dust, roughly independent of sky cut. A map of the spinning dust component from this fit and its error is shown in Figure 20. The morphology lies somewhat between that of dust and free-free emission, though the details depend on the specifics of the model. The Galactic plane is equally well fit by adding a synchrotron steepening parameter $c$ to the fit. The actual value of $c$ is generally not well constrained, but the average value in the plane is -1.8 . This very rapid steepening does not appear to be consistent with cosmic ray models (Strong et al. 2007), but may have some other physical origin.

Since the goodness of fit outside the plane is not improved by the addition of a spinning dust component, and low $\mathrm{S} / \mathrm{N}$ regions bias the spinning dust fraction upwards, we regard the spinning dust fraction of the fits above as an upper bound to the overall amount of diffuse spinning dust emission present. As with previous $W M A P$ fits, this new fitting technique continues to find that spinning dust is a subdominant emission process. 

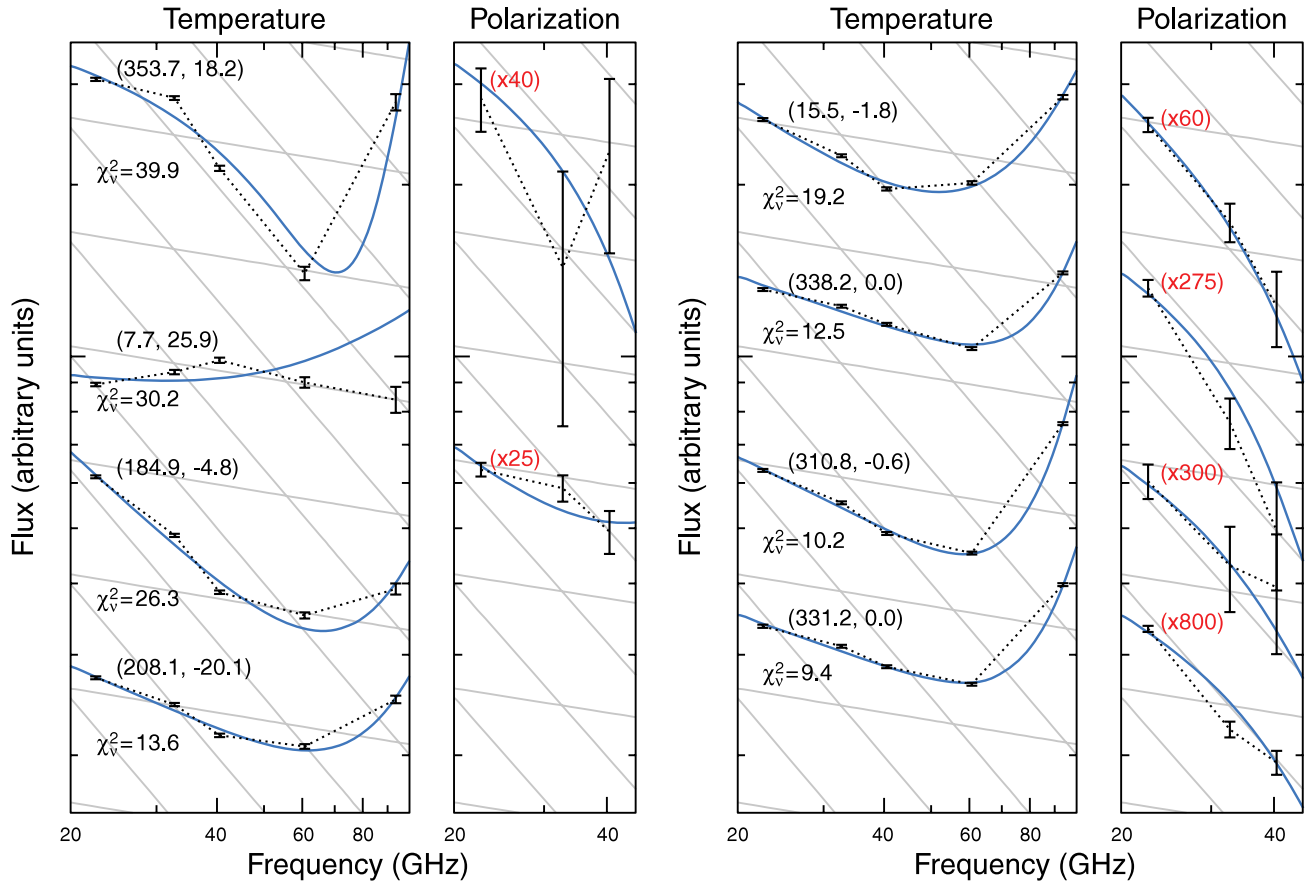

Figure 17. Temperature (Stokes $I$ ) and polarization $\left(\sqrt{Q^{2}+U^{2}}\right)$ spectra for poorly fit pixels in different regions of the sky. The vertical scale is arbitrary flux units, but polarization data are shown beside the corresponding temperature data, with a number in red indicating the approximate (K-band) ratio of intensity to polarization. Polarization data are not shown for pixels with low S/N. Numbers in parentheses are Galactic coordinates. The blue curve is the best-fit "base" model. The model does not include synchrotron steepening; convex slopes are due to negative CMB contributions. The grid of gray lines indicates spectral indices of $\alpha=-0.14$ and $\alpha=-1$. The left plot shows pixels further from the plane; the right plot shows pixels near the plane. The fit converged for all regions shown and none were covered by the point-source mask. However, the four pixels shown on the left are (from top to bottom) within a few degrees of $\rho$ Oph, $\zeta$ Oph, Tau A, and the Orion nebula.

\section{DISCUSSION}

\subsection{Effect on CMB and Cosmology}

The uncertainties of the fit in the Galactic plane preclude $\mathrm{CMB}$ analysis for those regions. Fortunately, such regions appear to be tightly confined to the plane inside a very narrow sky cut ( $9 \%$ of the total sky) and, thus, can be excluded without losing much information for cosmological analysis. The foreground maps from the MCMC fit are similar to those from the MEM fit and other foreground templates, which means that CMB polarization maps cleaned by using such templates will also be similar.

Outside of a narrow band on the Galactic plane, the CMB map produced by the fit is visually identical to the ILC map. The difference between the "base" fit CMB map and the ILC map is shown in Figure 21. The total variance of this difference map outside of the KQ85 mask used for power spectrum analysis is $116 \mu \mathrm{K}^{2}$, much lower than the CMB power. The variance between the "base" fit CMB map and the "shifted sd" CMB map is $44.1 \mu \mathrm{K}^{2}$ outside of the KQ85 mask; variance from one fit to another is generally even smaller for other combinations. Spherical harmonic decomposition did not show these total variances to be strongly focused on any particular multipole, and the numbers are small enough such that differences between maps fall within cosmic variance.

The CMB polarization maps produced by the MCMC fit presented in this work are noisy and show some evidence of synchrotron contamination. Nevertheless, the covariance maps from the fits can be used to bound the amount of contamination present, and are available on the LAMBDA Web site. These are produced from the (marginalized) variance of each parameter over the Markov chain for each pixel. For cosmological analysis, a different method is used to marginalize over polarization foregrounds. For a full description, see Dunkley et al. (2009).

\subsection{More Complicated Models}

So far, all of the models fit have assumed that the spectral shape of foreground emission in a $\sim 1 \mathrm{deg}^{2}$ pixel can be described as a sum of power laws or other simple shapes. This is justified if the observed emission is dominated by a few emission mechanisms, which simply combine additively along the LOS and have minor spatial variation within the beam. In more complex regions of the Galaxy, however, things may not be so simple.

If two synchrotron regions along the LOS have their polarization angles oriented nearly orthogonally, then the total polarized emission will be sharply reduced. If the two regions have different spectral indices, then the cancellation in polarization will be maximized at the frequency where the individual polarization amplitudes match, causing a dip in the polarization spectrum. Thus, even with pure synchrotron emission, the polarization spectrum can look quite different from the temperature spectrum.

To assess this effect, 100,000 Monte Carlo realizations were made of superposition of two independent randomly oriented synchrotron emitting regions. Parameters for the distribution of intensity and spectral index were chosen to roughly correspond to observations, but the simulation was meant only to provide a generous estimate of how different temperature and polarization behavior could be simply due to multiple synchrotron regions along the LOS. The mean spectral index difference was small $(-0.051)$ but the standard deviation was not insignificant (0.12) and the distribution was non-Gaussian with high kurtosis (4.4). Over $20 \%$ of the simulations had an absolute slope 

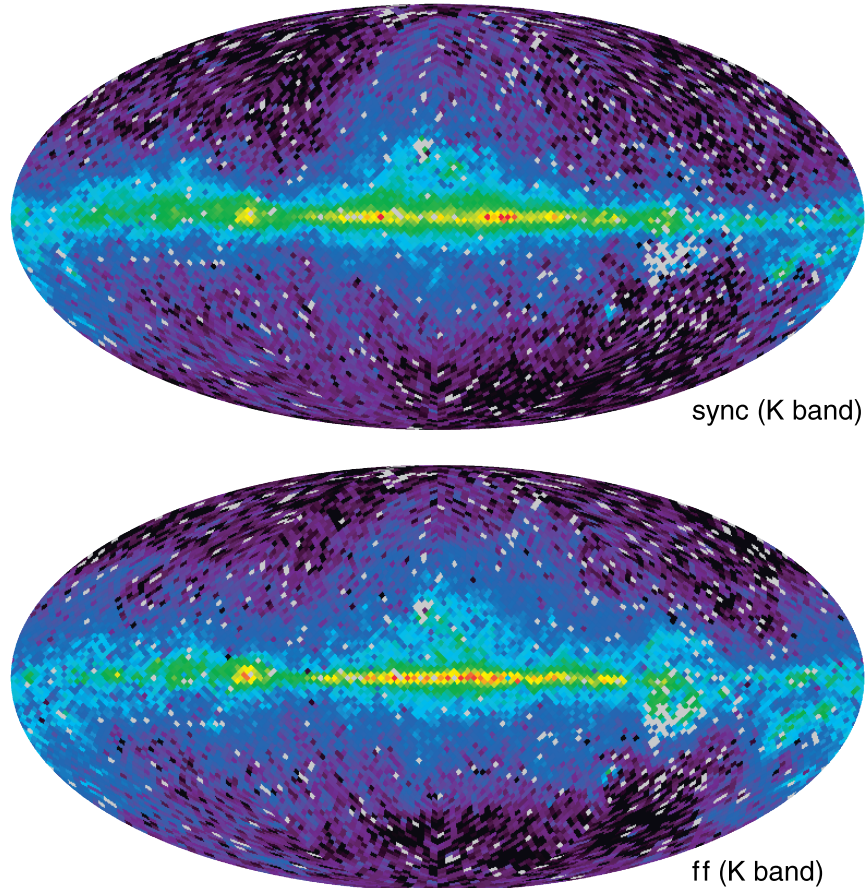

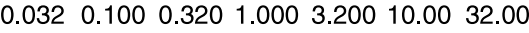
$\mathrm{T}(\mathrm{mK})$

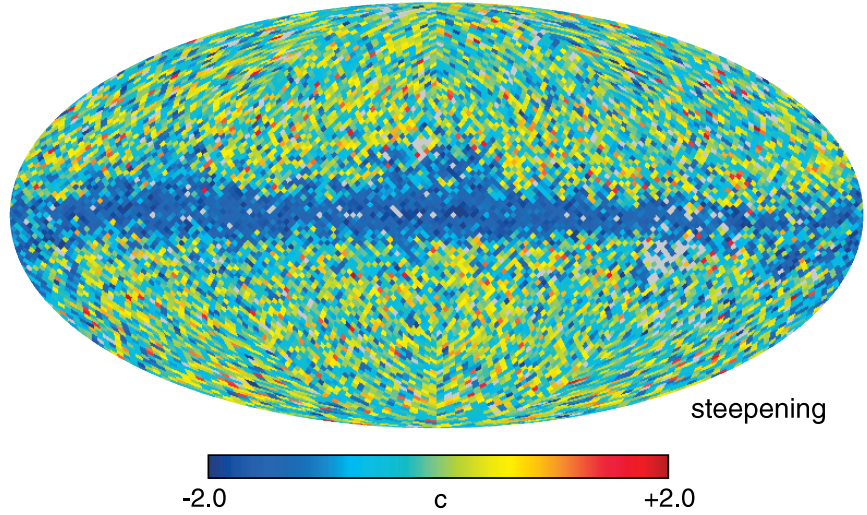

Figure 18. Maps for foreground components as determined by the MCMC fitting process for the "steep" model. This model has a frequency-dependent synchrotron spectral index $\beta_{s}(\nu)=\beta_{s}+c \ln \left(\nu / \nu_{K}\right)$. Synchrotron and free-free temperatures are measured at the $K$ band, and gray pixels are those masked due to point sources or flagged as problematic. Since this model does not differ from the "base" model at high frequencies, the thermal dust emission is unaffected and is not shown. Note that the steepening parameter tends toward large negative values in the Galactic plane. Top: synchrotron, middle: free-free, bottom: steepening parameter $c$.

difference larger than 0.1 . Thus, we cautiously conclude that while differences between temperature and polarization spectral indices at the $\sim 0.1$ level could be quite mundane in origin, consistent differences of 0.25 or larger are probably not due to chance alignments in the polarization angle and may be caused by an unpolarized nonthermal temperature component.

For the fit, free-free emission was modeled as a pure power law based on the assumption that the plasma is optically thin. In reality, $\mathrm{H}$ II regions can become dense enough to become optically thick at frequencies as high as $20 \mathrm{GHz}$, although such regions are spatially small and do not significantly contribute to the observed emission for a beam as large as WMAP's. Further, to obtain rising flux at the $\mathrm{K}$ band requires a very high emission measure $\left(\sim 10^{9} \mathrm{~cm}^{-6} \mathrm{pc}\right)$. Even if they were somehow significant, such regions cannot explain a steepening spectrum past the Ka band.

Synchrotron self-absorption can also cause a low-frequency turnover, but the physical parameters necessary for the turnover frequency to lie in or near the WMAP range imply conditions, typically, only found in active galactic nuclei (AGNs) or other extreme regions. It may be physically possible for synchrotron radiation from stellar-mass black hole jets or accretion disks to become optically thick at WMAP frequencies, but this phenomenon has yet to be clearly observed and it is unlikely that such emission would significantly contribute at WMAP's resolution.

Synchrotron radiation is suppressed when emitted from a region with a refractive index less than unity, such as a plasma. This is known as the Tsytovitch-Razin effect, and causes strong suppression of synchrotron emission below $20\left(n_{e} / B\right) \mathrm{Hz}$, where $n_{e}$ is the electron density (in $\mathrm{cm}^{-3}$ ) and $B$ is the perpendicular component of the magnetic field (in $\mathrm{G}$ ). For typical Galactic electron densities and magnetic fields, this cutoff is in the 3-300 $\mathrm{MHz}$ range, at most. Unless energetic electrons play an unexpectedly large role in diffuse emission, WMAP should not see significant Tsytovitch-Razin suppression.

Diffusive synchrotron radiation (DSR) differs from an ideal synchrotron spectrum because of the presence of significant random fluctuations in the magnetic field (Fleishman 2005). In this model, lower-energy electrons experience small-scale turbulence in the magnetic field structure and follow noncircular paths due to the random deflections. In such models, the emission spectrum can turn over from a power law with $\beta \approx-2.1$ in the turbulence-dominated diffusive regime to a normal synchrotron spectrum at higher frequencies. While in most models this occurs at low frequencies, there is some indication that for pulsar wind nebula, the turbulence is relevant up to the $\mathrm{GHz}$ range and above (Fleishman \& Bietenholz 2007). Whether DSR can occur for less compact objects is not understood at this time.

\subsection{Other Components}

Much has been written on the possible presence of anomalous emission in the lower frequency bands of WMAP. There are at least two categories of observations: one is of emission that is diffuse and significant over large portions of the sky outside the Galactic plane, and another where the emission is important and perhaps even dominant in specific compact regions.

Much evidence for diffuse anomalous emission comes from template correlations (de Oliveira-Costa et al. 1999; Bonaldi et al. 2007; Dobler \& Finkbeiner 2008) rather than direct fitting of the data (though for a recent example of the latter, see MivilleDeschenes et al. 2008). Characterizing the error and offsets in templates made from data at very different frequencies has proven challenging. Nevertheless, Dobler \& Finkbeiner (2008) showed that using an $\mathrm{H} \alpha$ template to fit WMAP data results in an improvement of the $\chi_{v}^{2}$ by 0.016 (to 2.977), and that the spectrum has a significant "bump." Though the improvement seems small, due to the large number of DOF, it is statistically significant, and appears to be robust against the systematic error investigated in that work. The Ka-band excess for select Galactic plane pixels (Figure 17), however, is in the data alone, independent of any template. Boughn \& Pober (2007) also found, by combining $19 \mathrm{GHz}$ data with WMAP K-band, that the Galactic plane seems to have antenna temperature falling less steeply than $\beta=-2$ (i.e., a rising flux spectrum). 

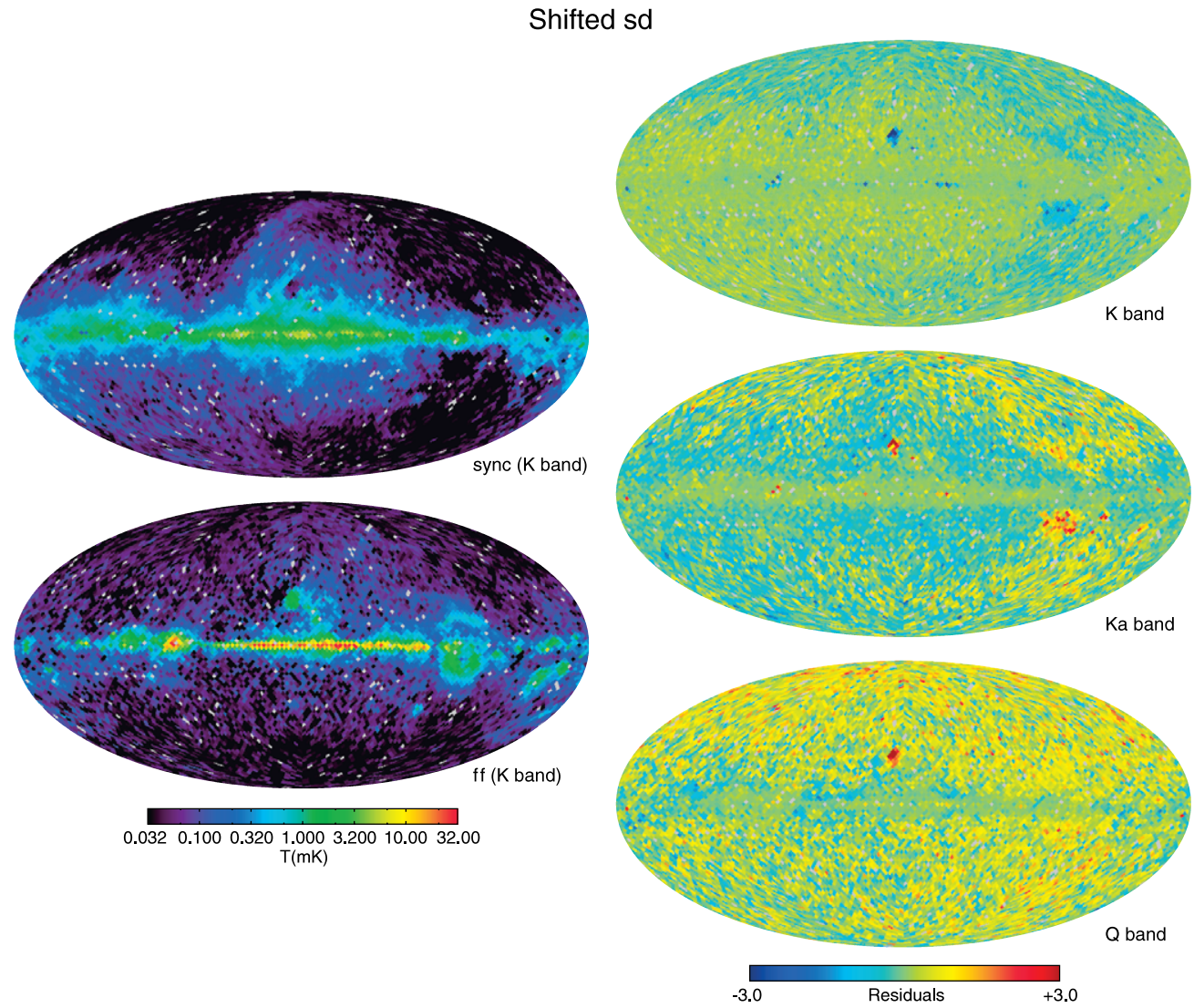

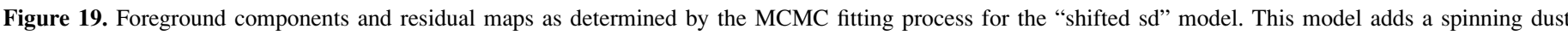

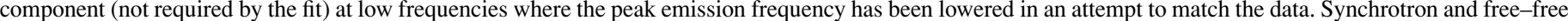

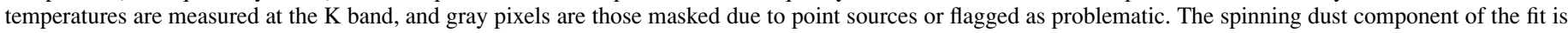

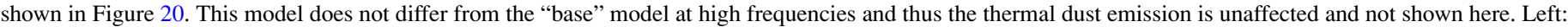
synchrotron and free-free maps, right: residuals (in dimensionless units of noise sigma) to the fit at K, Ka, and Q bands.

Specifically regarding compact regions, Finkbeiner et al. (2002) previously reported on two regions which might show excess emission in the $10-40 \mathrm{GHz}$ range due to spinning dust. CBI observations (Dickinson et al. 2006) failed to find anomalous emission from one, LPH96 201.663+1.643; other authors (McCullough \& Chen 2002) had previously raised the possibility that such emission might be due to an optically thick ultracompact $\mathrm{H}$ II region. The other, LDN 1622, was found by Casassus et al. (2006) to have a spectral energy distribution consistent with spinning dust, a result driven in part by the lack of flux at $5 \mathrm{GHz}$ found by the Parkes-MIT-NRAO survey of Condon et al. (1993). Other surveys at lower frequencies with a larger angular resolution, more comparable to WMAP's, however, have not measured a lack of flux - the $408 \mathrm{MHz}$ data summarized by Haslam et al. (1981) measured more flux than the WMAP $\mathrm{K}$ band and was consistent with a mixture of power-law thermal and nonthermal components for the region containing LDN 1622 .

Separately, Scaife et al. (2008) recently observed a sample of northern $\mathrm{H}$ II regions and found no evidence for anomalous emission in any, but observations with the Very Small Array (Scaife et al. 2007) find some evidence for a $33 \mathrm{GHz}$ excess in SNR 3C396. Thus, for compact regions, the status of anomalous emission appears to be mixed.

There has been some discussion in the literature of correlation between CMB maps and neutral hydrogen (Verschuur 2007), but this result was not found to be statistically significant (Land \& Slosar 2007).

\subsection{Directions for the Future}

It is quite probable that at least one of the above model complications or additional components is relevant for understanding our Galaxy. More data are needed, particularly in the $5-30 \mathrm{GHz}$ range. Further, the inability to measure flux at large angular scales is a problem for many observations, particularly when the angular scale limit depends on the observing frequency. This continues to make precise comparison of results difficult. Large-scale observations with calibration errors at the percent level or better are needed to address the nature of features seen in some pixels of the Galactic plane.

For the WMAP foreground fits, the dust spectrum was treated as a pure power law. In reality, dust emission in the WMAP bands is probably dominated by a cold component with a low enough temperature that the exponential cutoff is not negligible. However, since the frequency range of WMAP's dust sensitivity is narrow, the largest effect of the exponential correction is simply a modification of the apparent power-law index, which, for typical cold dust temperatures $(\sim 10 \mathrm{~K})$, amounts to a change of about 0.1 in $\beta_{d}$. This effective bias is, therefore, small compared to typical errorbars. However, there are already hints that the extrapolation of dust models to millimeter wavelengths is not entirely satisfactory.

Further insight into Galactic foregrounds will be obtained from upcoming experiments. For example, the Planck satellite (Tauber 2005), scheduled to be launched in 2008, will soon provide more insight into Galactic foregrounds. While Planck's frequency coverage does not extend low enough to overlap 

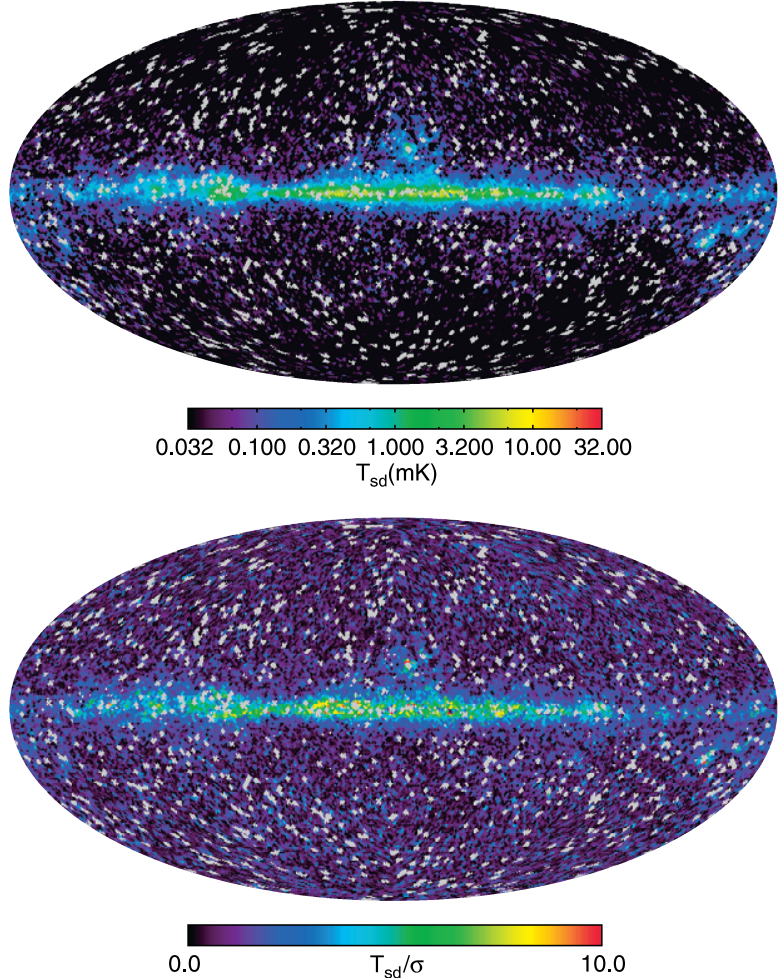

Figure 20. Top: map of possible spinning dust antenna temperature $T_{s d}$ (as measured at the $\mathrm{K}$ band) for the "shifted" spinning dust model fit. While the model is based on a spinning dust spectrum, the data are insufficient for distinguishing the physical emission mechanism from other possibilities mentioned in the text. Maps of the other three foregrounds are qualitatively similar to that of the "base" model, with the synchrotron amplitude reduced somewhat to accommodate the additional low-frequency component. Bottom: map of spinning dust temperature divided by the marginalized temperature error as calculated by the MCMC chain. This is statistical error only and does not include uncertainties in the model.
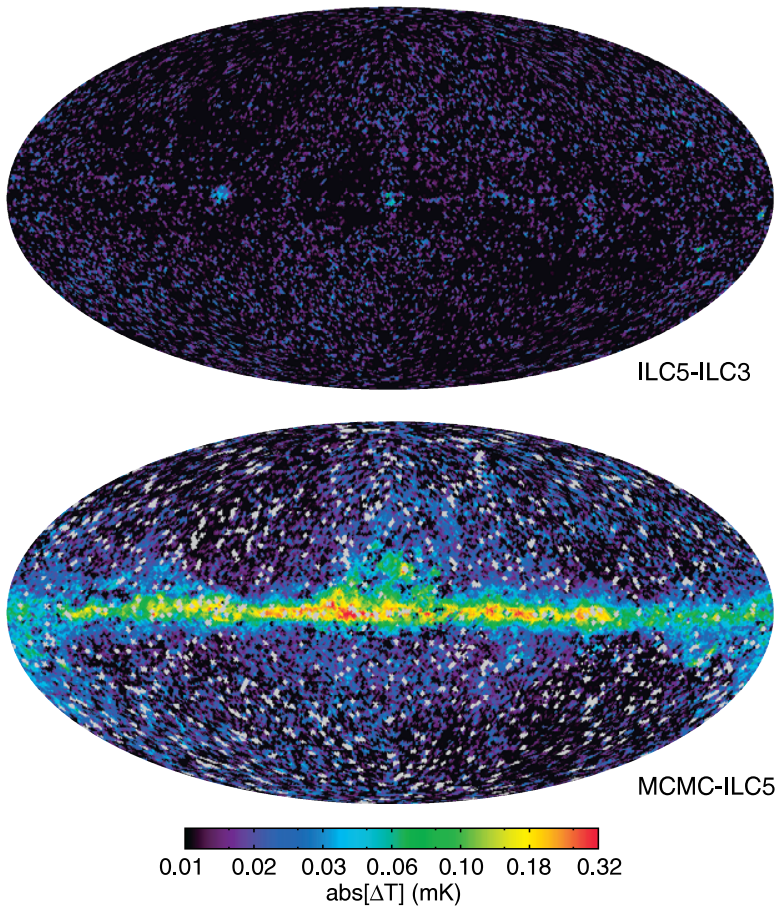

Figure 21. Difference maps between the 5 year and 3 year ILC maps (top), and the MCMC fit for the CMB and the 5 year ILC map (bottom). For the former, the most prominent large-scale difference is from the reduction of noise with WMAP's observational pattern. Even for the latter, the variance outside the $\mathrm{KQ} 85$ analysis mask is $116 \mu \mathrm{K}^{2}$, lower than the CMB power at large scales.
WMAP's K band, Planck will observe at WMAP's other frequencies with roughly $25 \%$ narrower beams and an order-ofmagnitude better sensitivity. Comparison to WMAP's results will be an important check of systematic errors, and the increased sensitivity can help with foreground discrimination, for example by improving knowledge of the spectral index for polarized synchrotron emission. Further, Planck has six higher frequency channels in the $100-860 \mathrm{GHz}$ range, which will be invaluable for studying dust to a precision several orders of magnitude better than what was available with prior data in this frequency range.

\section{CONCLUSIONS}

1. WMAP's temperature and polarization data outside the Galactic plane are well described by the standard three foreground components: synchrotron, free-free, and thermal dust, each with power-law spectral indices.

2. The spectral index for synchrotron radiation at high latitudes is consistent with $\beta \approx-3$, with a trend toward $\beta=-2.7$ seen at lower latitudes. The spectral index for dust is not well constrained but appears consistent with $\beta \cong 2$.

3. Some localized regions in the Galactic plane show emission with $\beta \approx-2$ below $33 \mathrm{GHz}$, which steepens by as much as $\Delta \beta=-0.8$ above $33 \mathrm{GHz}$, and this emission is mostly unpolarized. Both spinning dust and synchrotron steepening models can be used to fit this emission component, whose physical origin is unclear.

4. CMB maps from different model fits show $<50 \mu \mathrm{K}^{2}$ of variance relative to each other outside the KQ85 analysis mask, and $<120 \mu \mathrm{K}^{2}$ of variance relative to the 5 year ILC map. The CMB and cosmological results are robust to changes in the foreground model.

5. WMAP serves as a precise ( $<1 \%$ error), unbiased, fullsky survey of the Galaxy, which can reveal large-scale microwave emission features that were never seen before.

The WMAP mission is made possible by the support of the Science Mission Directorate Office at NASA Headquarters. This research was additionally supported by NASA grants NNG05GE76G, NNX07AL75G S01, LTSA03-0000090, ATPNNG04GK55G, and ADP03-0000-092. This research has made use of NASA's Astrophysics Data System Bibliographic Services. We acknowledge use of the HEALPix, CAMB, and CMBFAST packages.

\section{REFERENCES}

Agladze, N. I., Sievers, A. I., Jones, S. A., Burlitch, I. M., \& Beckwith, S. V. W. 1994, Nature, 372, 243

Bennett, C. L., et al. 2003, ApJS, 148, 97

Bonaldi, A., Ricciardi, S., Leach, S., Stivoli, F., Baccigalupi, C., \& de Zotti, G. 2007, MNRAS, 382, 1791

Boughn, S. P., \& Pober, J. C. 2007, ApJ, 661, 938

Casassus, S., Cabrera, G. F., Förster, F., Pearson, T. J., Readhead, A. C. S., \& Dickinson, C. 2006, ApJ, 639, 951

Condon, J. J., Griffith, M. R., \& Wright, A. E. 1993, AJ, 106, 1095

Cornwell, T., Braun, R., \& Briggs, D. S. 1999, in ASP Conf. Ser. 180, Synthesis Imaging in Radio Astronomy II, ed. G. B. Taylor, C. L. Carilli, \& R. A. Perley (San Francisco, CA: ASP), 151

de Oliveira-Costa, A., et al. 1999, ApJ, 527, L9

Dickinson, C., Casassus, S., Pineda, J. L., Pearson, T. J., Readhead, A. C. S., \& Davies, R. D. 2006, ApJ, 643, L111

Dobler, G., \& Finkbeiner, D. P. 2008, ApJ, 680, 1222

Draine, B. T., \& Lazarian, A. 1998, ApJ, 494, L19 
Dunkley, J., Bucher, M., Ferreira, P. G., Moodley, K., \& Skordis, C. 2005 MNRAS, 356, 925

Dunkley, J., et al. 2009, ApJS, 180, 306

Eriksen, H. K., Dickinson, C., Jewell, J. B., Banday, A. J., Gorski, K. M., \& Lawrence, C. R. 2008, ApJ, 672, L87

Finkbeiner, D. P. 2003, ApJS, 146, 407

Finkbeiner, D. P., Davis, M., \& Schlegel, D. J. 1999, ApJ, 524, 867

Finkbeiner, D. P., Schlegel, D. J., Frank, C., \& Heiles, C. 2002, ApJ, 566, 898

Fleishman, G. D. 2005, in Lecture Notes in Physics 687, Geospace Electromagnetic Waves and Radiation, ed. J. W. Labelle \& R. A. Treumann (Berlin: Springer), 87

Fleishman, G. D., \& Bietenholz, M. F. 2007, MNRAS, 376, 625

Gilks, W. R., Richardson, S., \& Spiegelhalter, D. J. 1996, Markov Chain Monte Carlo in Practice (London: Chapman and Hall)

Gorski, K. M., Hivon, E., Banday, A. J., Wandelt, B. D., Hansen, F. K., Reinecke, M., \& Bartlemann, M. 2005, ApJ, 622, 759

Green, D. A. 1988, Ap\&SS, 148, 3

Green, D. A., \& Scheuer, P. A. G. 1992, MNRAS, 258, 833

Haslam, C. G. T., Klein, U., Salter, C. J., Stoffel, H., Wilson, W. E., Cleary, M. N., Cooke, D. J., \& Thomasson, P. 1981, A\&A, 100, 209

Haslam, C. G. T., Stoffel, H., Salter, C. J., \& Wilson, W. E. 1982, A\&AS, 47, 1

Hill, R., et al. 2009, ApJS, 180, 246

Hinshaw, G., et al. 2007, ApJS, 170, 288

Hinshaw, G., et al. 2009, ApJS, 180, 225

Hummel, E., Dahlem, M., van der Hulst, J. M., \& Sukumar, S. 1991, A\&A, 246, 10

Itoh, N., Sakamoto, T., Kusano, S., Nozawa, S., \& Kohyama, Y. 2000, ApJS, 128,125

Kogut, A., et al. 2007, ApJ, 665, 355

Kunz, M., Trotta, R., \& Parkinson, D. R. 2006, Phys. Rev. D, 74, 023503
Land, K., \& Slosar, A. 2007, Phys. Rev. D, 76, 087301

Lawson, K. D., Mayer, C. J., Osborne, J. L., \& Parkinson, M. L. 1987, MNRAS, 225, 307

McCullough, P. R., \& Chen, R. R. 2002, ApJ, 566, L45

Meny, C., Gromov, V., Boudet, N., Bernard, J.-P., Paradis, D., \& Nayral, C. 2007, A\&A, 468, 171

Miville-Deschenes, M., Ysard, N., Lavabre, A., Ponthieu, N., Macias-Perez, J. F., Aumont, J., \& Bernard, J. P. 2008, A\&A, 490, 1093

Oster, L. 1961, ApJ, 134, 1010

Page, L., et al. 2003, ApJS, 148, 39

Page, L., et al. 2007, ApJS, 170, 335

Press, W. H., Teukolsky, S. A., Vetterling, W. T., \& Flannery, B. P. 1992 Numerical Recipes in C (2nd ed.; Cambridge: Cambridge Univ. Press)

Quireza, C., Rood, R. T., Bania, T. M., Balser, D. S., \& Maciel, W. J. 2006, ApJ, 653, 1226

Reich, P., \& Reich, W. 1988, A\&AS, 74, 7

Reich, P., Reich, W., \& Testori, J. C. 2004, in The Magnetized Interstellar Medium, ed. B. Uyaniker, W. Reich, \& R. Wielebinski (Katlenburg-Lindau: Copernicus $\mathrm{GmbH}$ ), 63

Scaife, A., et al. 2007, MNRAS, 377, L69

Scaife, A. M. M., et al. 2008, MNRAS, 385, 809

Schlegel, D. J., Finkbeiner, D. P., \& Davis, M. 1998, ApJ, 500, 525

Strong, A. W., Moskalenko, I. V., \& Ptuskin, V. S. 2007, Annu. Rev. Nucl. Part. Sci., 57, 285

Tauber, J. A. 2005, in IAU Symposium 201, New Cosmological Data and the Values of the Fundamental Parameters, ed. A. N. Lasenby \& A. Wilkinson (San Francisco, CA: ASP), 86

Verschuur, G. L. 2007, ApJ, 671, 447

Wright, E. L., et al. 2009, ApJS, 180, 283 